\title{
VIBRATIONS IN A 7-ROD BUNDLE SUBJECT TO AXIAL FLOW: SIMULATIONS AND EXPERIMENTS
}

\author{
H. Dolfen ${ }^{\mathrm{a}, *}$, F. Bertocchi ${ }^{\mathrm{b}}$, M. Rohde ${ }^{\mathrm{b}}$, J. Degroote ${ }^{\mathrm{a}, \mathrm{c}}$ \\ ${ }^{a}$ Department of Flow, Heat and Combustion Mechanics, Ghent University, Sint-Pietersnieuwstraat 41, B-9000 \\ Ghent, Belgium, E-mail: henri.dolfen@ugent.be \\ ${ }^{b}$ Department of Radiation Science and Technology, Delft University of Technology, Mekelweg 15, 2629JB \\ Delft, The Netherlands \\ ${ }^{\mathrm{c}}$ Flanders Make, Belgium
}

\begin{abstract}
Being able to quantify mechanical vibrations is of key importance for the safety of nuclear power plants, as they are able to induce damage. In this work, numerical simulations are used to compute water flow and vibration in a densely packed bundle of 7 rods, mimicking an experimental setup. This flow configuration is chosen to resemble the coolant flow through a nuclear reactor core. Because of the wall proximity, a considerable velocity difference between the narrow gaps and the subchannels exists, with an inflection point in the velocity profile. This yields an unstable situation, and large vortices are continuously created through a mechanism similar to the Kelvin-Helmholtz instability. The vortex streets in between the rods are associated with a fluctuating pressure field, causing vibrations of the rods.

The experimental setup contains 7 steel cylinders, encased in a hexagonal duct. The central rod contains a section where the steel is replaced by a water-filled silicone tube, clamped at both extremes to the steel rod, and the vibrations of this section are examined. The numerical approach consists of coupled fluid-structure interaction (FSI) simulations, with the flow being modelled using computational fluid dynamics (CFD) and the structure using computational solid mechanics (CSM).

The available experimental data consist of Laser Doppler Anemometry (LDA) measurements and high-speed camera footage of the wall movement of the silicone rod. Equivalent data is collected from the numerical simulations. The simulations are repeated for different flow rates. The frequency spectrum of the coherent structures, and the frequency and amplitude of the wall movement are compared for each operating point, as well as their trend as a function of the flow rate. The dominant frequencies found in the simulation results were similar to the experimental results, although slightly higher. They also showed a linear trend, just like the experiments. A larger mismatch was present for the structural response, the frequencies found using the FSI model being more than twice as high.
\end{abstract}

\section{INTRODUCTION}

Tube bundles are often encountered in nuclear reactor cores and heat exchangers. This kind of geometry placed in fluid flow is subjected to loads that can trigger vibrations. Being able to quantify these vibrations is of key importance for the safe operation of nuclear power plants, so they have to be considered during the design process. Several mechanisms exist that trigger vibrations, as it will be explained in the following paragraphs.

It is well known that long, elastic tubes placed in high-velocity axial flow can display fluttering and buckling phenomena due to fluid-elastic instabilities (Païdoussis, 2004). It has been acknowledged that this source of vibration can be harmful, causing noise in the best case, but destructive damage in the worst.

Another mechanism that could be present is vibration due to a variable load present in the fluid. An obvious source of variability is turbulence, with which a chaotic, fluctuating pressure field is associated. So-called turbulence-induced vibration can arise, which has a small amplitude. Analytical work towards this has been done by Païdoussis and Curling (1985), who expressed the vibration response to an incident turbulent axial flow. This work was followed by experiments, also by Curling and Païdoussis (1992a, 1992b). Numerical work has been carried out too. Large-eddy simulations (LES) are suitable, as they are able to resolve the turbulent structures, unlike Reynolds-averaged Navier-Stokes (RANS) methods, while requiring less computing power than direct numerical simulations (DNS). An example of recent research using LES is the work of De Ridder et al. (2016a).

The main mechanism of interest here is vibration due to large-scale coherent structures. In a tube bundle geometry, different regions can be distinguished based on the flow through area. The regions where there is proximity of the walls, like the gap between two rods, are for obvious reason called the 'gap regions'. The other regions, farther away from the walls are called 'subchannels'. These locations are indicated on Figure 1. A gap region has a lower hydraulic diameter than a subchannel region. Because of this, a substantial velocity difference exists between the two. In the shear layer between the two regions, flow pulsations emerge. The velocity profile along a line connecting a gap and a subchannel contains an inflection point. This results in an instability very similar to the Kelvin-Helmholtz instability, which often occurs in shear layers. For instance, from meteorology this is known to happen in the atmosphere where two air masses of different speed meet. A street of vortices arises and parcels of fluid cross the shear layer back and forth between gap and subchannel. This phenomenon is strongest for densely packed rod bundles, where the gaps are small enough to have an important velocity discrepancy, but not so small that no cross-flow can occur (De Ridder et al., 2016b). This results in increased mixing rates, which cannot be explained by turbulent diffusion alone (Meyer, 2010). The increased mixing rate also enhances the heat transfer, which is of interest for the design of nuclear reactor cores (Chang and Tavoularis, 2006). A comprehensive overview of the research towards this flow phenomenon is given by Meyer (2010). A contribution of Tavoularis (2011) attempts to come to a unified nomenclature, and gives an overview of different methods. The term 'gap vortex street' is 
proposed. Experimental research has, among others, been executed by Hooper and Rehme (1984), Meyer and Rehme (1994) and Choueiri and Tavoularis $(2014,2015)$ on a more fundamental level using simple geometries. A more complex geometry, a 5-rod model of a 37-rod CANDU reactor, was investigated by Baratto et al. (2006).

In the last two decades the use of numerical techniques has steadily increased. Among the research on simplified geometries is the work of Chang and Tavoularis (2005), who simulate the experiments of Guellouz and Tavoularis (2000a, 2000b) using a Reynolds Stress Model. Since a network of gaps and subchannels is present in a rod bundle, multiple vortex streets are present. This leads to a more chaotic and complex behaviour of the pulsating flow, showing strong correlation between the different gap vortex streets (Baratto et al., 2006), which suggests more complex simulations are necessary. This was addressed in the work of Chang and Tavoularis (2007). In their research the same geometry was simulated, although exploiting rotational symmetry by using periodic boundary conditions. Similar work has been done by De Ridder et al. (2016b), taking also into account fluid-structure interactions (FSI). Not only RANS models have been used; examples of recent works using LES are the articles of Merzari and Ninokata (2011) and De Moerloose et al. (2018), in which periodic boundaries were used effectively to moderate the computational cost.

In this research another aspect is also focused on. The gap vortex street entails a fluctuating pressure field, subjecting the tubes to vibrations. These can be called 'vortex-induced vibrations'. The term is shared with vibrating cylinders in cross-flow. There are however important differences: no vortices are shed from the structure, the coherent structures move parallel to the rods, and the vibration amplitude is lower. Despite the low amplitude, the vibrations can be harmful. Possible consequences are noise, fatigue and fretting damage. Note that the imposed load has a more harmonic and less chaotic nature compared to isotropic turbulence. In this work, an effort was made to create a numerical model that predicts the flow-induced vibration and validate it against experimental measurements. To achieve this, the most relevant features of an experimental setup are selected and simplified. A full fluid-structure interaction simulation was performed, coupling a computational fluid dynamics (CFD) model with a computational solid mechanics (CSM) model. Figure 1 shows a schematic of the tube bundle that is considered, consisting of 7 rods in a hexagonal duct. In the next section the experimental setup is discussed more in depth, followed by an explanation of the numerical methodology. The results are analysed and discussed in Section 4.

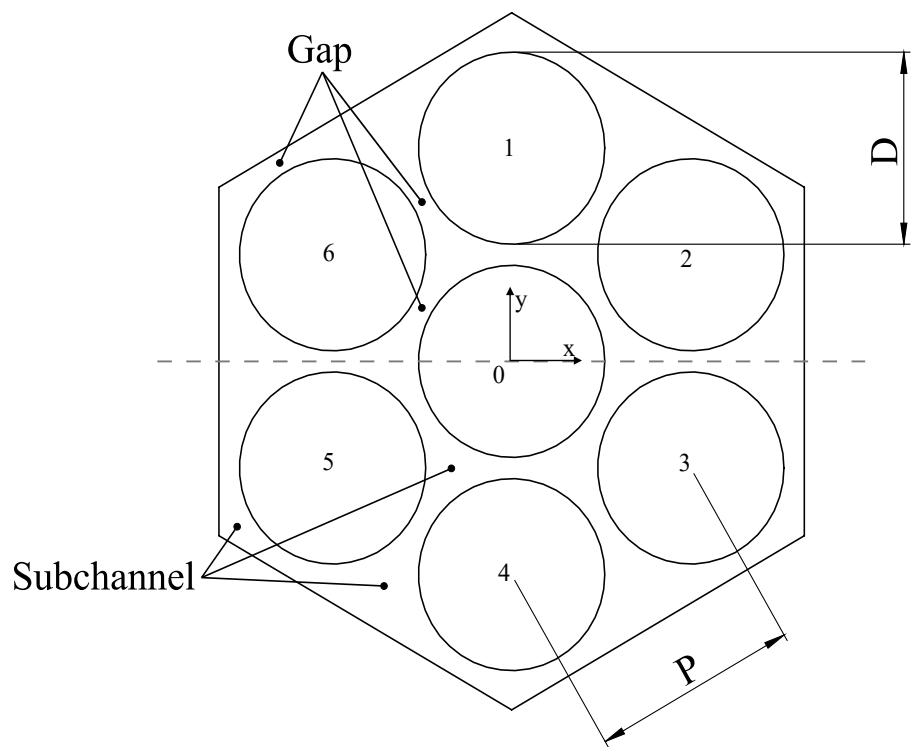

Figure 1. Schematic of the geometry under consideration with indication of the pitch (P) and diameter (D).

\section{EXPERIMENTAL SETUP}

The experimental setup (see Figure 2) is located at Delft University of Technology. It consists of 7 steel rods in a hexagonal lattice, placed in a hexagonal channel. The pitch-over-diameter or $\mathrm{P} / \mathrm{D}$ ratio is 1.11 , which is in the range where these flow pulsations have been observed. The assembly is placed vertically, and the flow is fed from a container located above. The water level in that container is controlled to assure a constant head, and thus constant flow rate. The flow is introduced via a diffusor, containing a grid to distribute the flow. Close to the bottom, a section is present in which the steel is replaced by transparent materials. The hexagonal duct is made of acrylic glass, while part of the six peripheral rod are made of fluorinated ethylene propylene (FEP). Those materials are chosen because their refractive index matches well with the one of water. Thanks to the optical access, Laser Doppler Anemometry (LDA) techniques can be used, as has been demonstrated on a predecessor of the experimental setup, investigating the same phenomenon and described in the work of Bertocchi et al. (2018). At the location with transparent duct and peripheral rods, a hollow silicone section is introduced in the central rod. It slides at the two extremes over the steel rod, such that a constant external diameter is maintained for the entire setup. This results in a $100 \mathrm{~mm}$ long section of the central rod that is flexible, and thus susceptible to vibrations. 


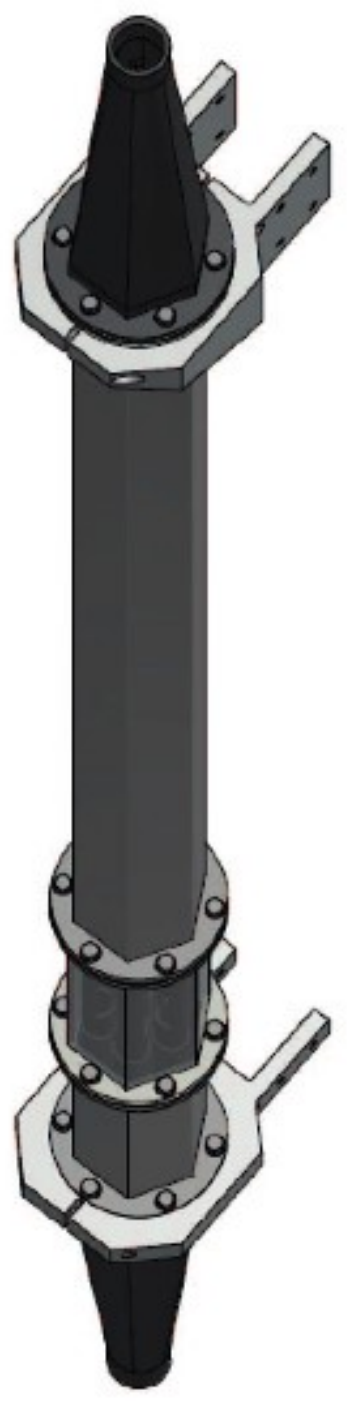

(a)

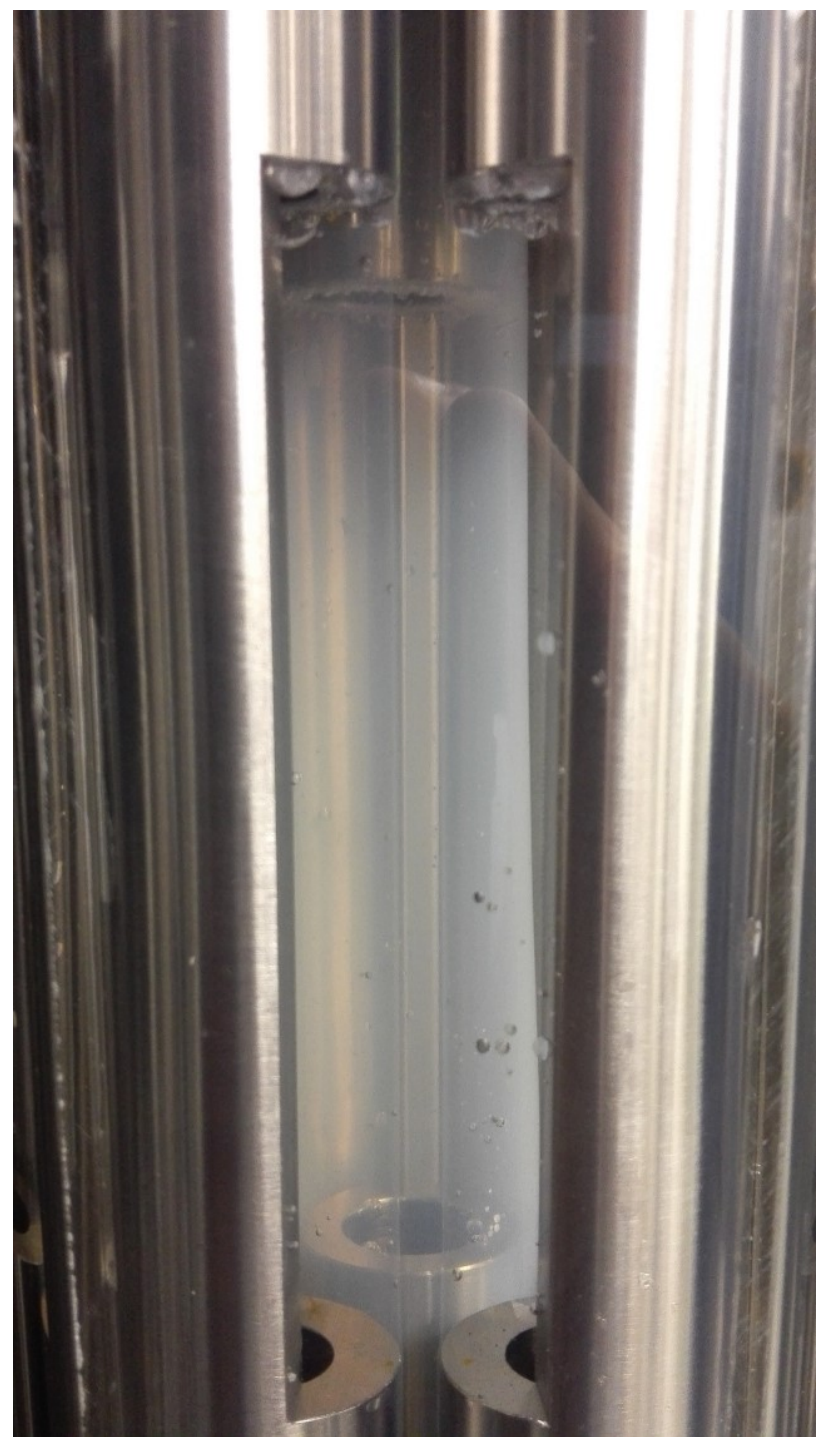

(b)

Figure 2. (a) Overview of the test section. (b) Close-up picture of the silicone rod illustrating the mounting and optical access.

A high-speed camera captures the vibrating silicone rod. The images were analysed and the position of the edge was determined based on the intensity. The sampling rate was $300 \mathrm{~Hz}$ and a filter served to remove erroneous outliers (due to air bubbles present in the flow). The range spanned between the maximal deflections is in the order of 10 pixels, so the displacement data is rather stepwise. The mean and root-mean-square (RMS) are calculated after converting from pixels to millimetres. The conversion factor is determined from a fixed reference with known length in the image. The spectrum was calculating using Bartlett's method (Monson, 1996) to reduce noise.

The dimensions and material properties are listed in Table 1. For a more detailed description of the experimental facility and measurement campaign, the reader is referred to Bertocchi et al. (2019).

Table 1. Overview of the domain dimensions and material properties.

\begin{tabular}{|l|l|l|}
\hline \multicolumn{2}{|c|}{ Dimensions } \\
\hline Rod diameter & $\mathrm{D}_{\text {out }}(\mathrm{m})$ & 0.03 \\
Pitch & $\mathrm{P}(\mathrm{m})$ & 0.0333 \\
Gap width & $\mathrm{G}(\mathrm{m})$ & 0.0033 \\
Flexible part length & $\mathrm{L}_{\text {flex }}(\mathrm{m})$ & 0.10 \\
\hline \multicolumn{2}{|c|}{ Material properties } & 995.10 \\
\hline Density of water & $\rho_{\text {water }}\left(\mathrm{kg} / \mathrm{m}^{3}\right)$ & $8.23 \times 10^{-4}$ \\
Dynamic viscosity of water & $\mu_{\text {water }}(\mathrm{Pa} \mathrm{s})$ & 1180.00 \\
Density of silicone & $\rho_{\text {silicone }}\left(\mathrm{kg} / \mathrm{m}^{3}\right)$ & $1.00 \times 10^{6}$ \\
Young's modulus of silicone & $E_{\text {silicone }}(\mathrm{Pa})$ & 0.47 \\
Poisson's ratio of silicone & $v_{\text {silicone }}$ & \\
\hline
\end{tabular}




\subsection{Fluid model}

The investigation was conducted in two stages. In a first stage, only the fluid dynamics were considered. A numerical model was created in ANSYS Fluent, which is a finite volume CFD code. The domain was created from a simplified version of the setup's geometry. It consists of a $1 \mathrm{~m}$ section of the setup, with the 7 rods and hexagonal duct modelled as no-slip walls. No other geometric features, like the diverging entrance part or grid spacers, were considered. A structured mesh was constructed, consisting of 120 cells along the circumference of each rod, 10 cells between the rods and 700 cells in axial direction, totalling 4200000 cells. A crosssection of the mesh is shown in Figure 3 a.

The front and back face were given a translational periodic boundary condition, such that the flow leaving at the back enters directly at the front. At this point, all exterior faces are assigned boundary conditions, yet there is nothing driving the flow, so a streamwise pressure gradient is added, which is implemented as a source term in the axial momentum equation. The simulation was performed repeatedly to determine the pressure gradient that produces a certain flow rate, with each simulation for the adjustment of the pressure gradient comprising 10000 time steps of $0.55 \mathrm{~ms}$. Based on the outcome of a simulation, a new guess for the source term was determined. This procedure was stopped when the achieved flow rate was within $1 \%$ of the desired one, which usually happened after 3-4 trials. The advantage of using periodic boundary conditions is that the flow is inherently developed. This allows using a rather short domain, moderating the computational effort. The chosen length is deemed sufficient, allowing enough wavelengths of the vortex street in the domain. By comparing frequency data at the middle of the silicone rod with data at the end and beginning, it was confirmed that in reality a fully developed flow is present in the test section, supporting the use of periodic boundary conditions.

An unsteady RANS (URANS) approach is followed, which, unlike RANS, is well able to capture the fluctuating flow as De Ridder et al. (2016b) state. More specifically, the k- $\omega$ SST turbulence model (Menter, 1994) is applied. Momentum and turbulent quantities are discretized using second order schemes. Time discretization is also done second order. In order to resolve well the fluctuating phenomena that occur, the time step size should be chosen sufficiently small. For the CFD part it was fixed on 0.55 ms and 20000 time steps were performed, resulting in a simulation time of $11 \mathrm{~s}$. In Section 4 it is validated that the time step size and simulation window are appropriately chosen. Velocity data is collected from these simulations, allowing to make the comparison with the LDA-measurements from the experiments.

\subsection{Fluid-structure interaction model}

In the second stage, a two way coupled FSI model produces the displacement data to compare to the experimental data. It is based on the CFD model previously discussed, although with some important adaptations. Also a finite elements model was created in the Abaqus software package. A partitioned approach was used to couple between fluid and structure, such that their respective models can be considered as black boxes. The Arbitrary Lagrangian-Eulerian (ALE) method (Donea, 2004) was employed to accommodate deformation of the CFD mesh.

Care has to be taken due to the periodic boundary conditions of the CFD domain. Its length is considered sufficiently long compared to the length of the vibrating structure, such that any perturbation of the flow due to the motion of the structure has decayed before reaching the structure again, after re-entering the domain through the periodic boundary. The model, further explained below, is schematically shown in Figure $3 \mathrm{~b}$ and Figure 3c.

The finite element model was built only for the part of the silicone rod that is able to move, approximating its connection to the metal rod by clamped boundary conditions at the two extremes. In reality it slides over the steel rod for a length of $50 \mathrm{~mm}$ at both sides, but this part of the silicone is considered entirely fixed. The modelled part has a length of $100 \mathrm{~mm}$. A mesh consisting of 6600 quadratic elements was constructed: 44 along the circumference, 50 axially and 3 in the thickness direction.

The silicone rod is not only subjected to the water flow surrounding it, but is also in contact with a column of water at its interior. This column is closed off at the bottom (right hand side of Figure 3c), but is at the top connected to the rest of the flow in such a way that only the static pressure is transmitted. The connection to the outer flow is simplified as being annular and a mesh interface is present where it connects to the outer domain, as the faces are not conformal. This fluid inside the central rod cannot be neglected in the FSI model, because its inertia could influence the vibration of the rod. Its total length is $1640 \mathrm{~mm}$ in reality but in the model it was chosen to reduce this to $800 \mathrm{~mm}$, because experience learned that otherwise convergence of the coupling iterations is deteriorated. This is believed to be due to the formation of a spurious pressure gradient in the first coupling iteration when the column of liquid shifts up or down to accommodate the change in internal volume of the tube. This pressure gradient does not belong to the converged solution but causes on its turn an unphysical deformation of the structural model. This behaviour deteriorates the solution and gets worse with increasing length. For the interior part, a relatively coarse mesh is applied, as no complex flow phenomena are expected. It only serves to accommodate a change of internal volume, in which case the fluid moves up or down a bit. Note that although the motion is small, a lot of mass is associated with it. The source term that drives the outer flow is disabled for this inner region. After adding the meshed inner domain (dark blue in Figure 3b) to the mesh described in Section 3.1, using an interface (purple in Figure 3b), an additional 5000 CFD-time steps are performed, allowing the flow to settle again after being slightly disturbed due to the topology change of the domain. 


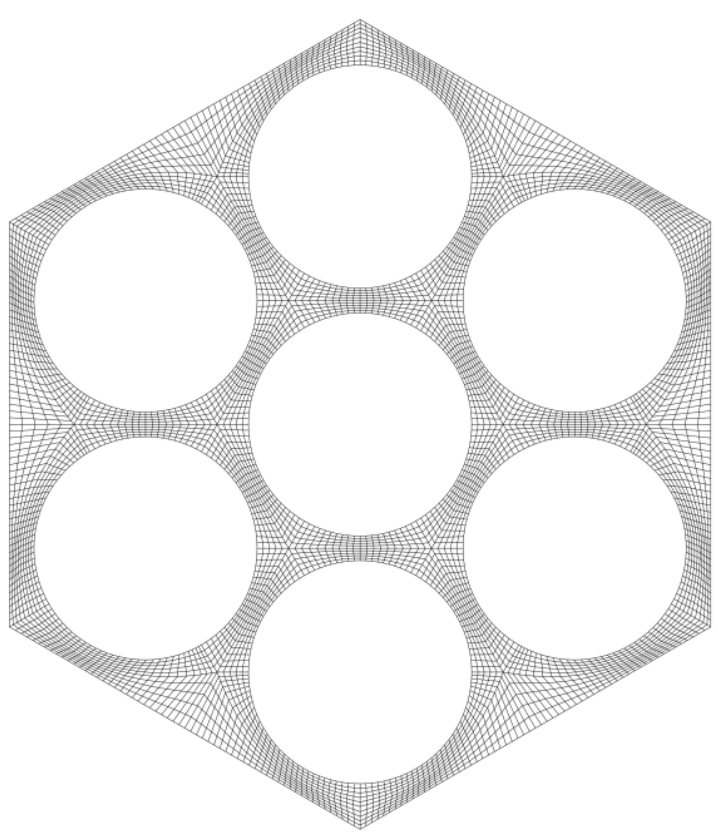

(a)

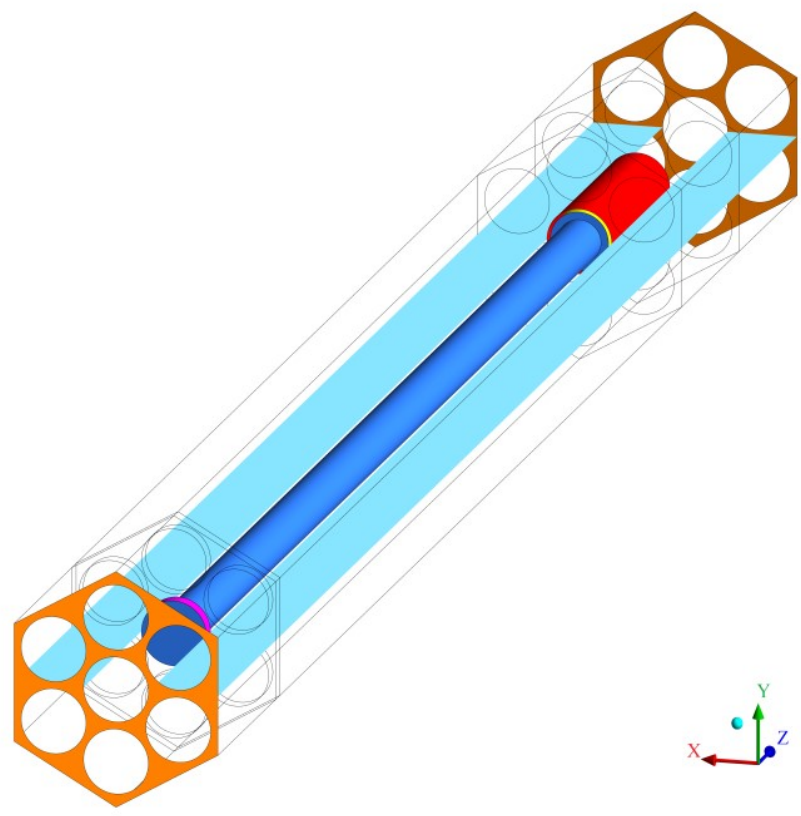

(b)

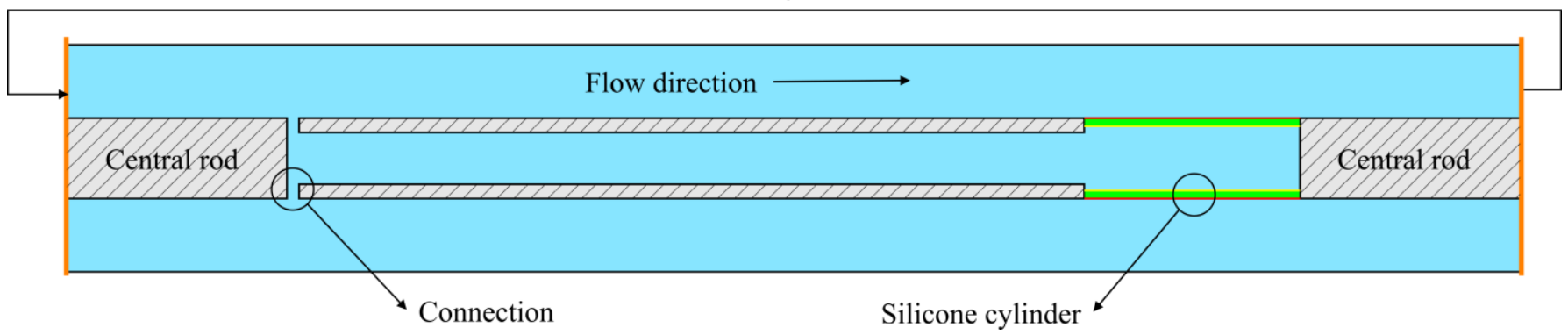

(c)

Figure 3. (a) Cross-section of the mesh. (b) The CFD domain after the inner part (dark blue) has been added. The periodic boundaries are shown in orange, the connection between the inner and outer domain in purple, the inner fluid-structure interaction surface in yellow and the outer fluid-structure interaction surface in red. The light blue plane corresponds with the dashed line in Figure 1. (c) Schematic of the FSI simulation domain (not to scale) in the plane indicated with a dashed line in Figure 1: crosssection in a plane in between peripheral rods and through the central rod, the fluid domain is indicated in light blue, the flexible part of the solid domain in green and the interfaces in red when connecting with the outer fluid domain and in yellow when connecting with the inner fluid domain. The periodic boundaries are again indicated in orange.

This configuration requires two concentric fluid-structure interfaces. One is the contact surface between the outer flow and the structure (red in Figure $3 \mathrm{~b}$ and Figure $3 \mathrm{c}$ ), and the other one between the structure and inner fluid domain (yellow in Figure $3 \mathrm{~b}$ and Figure 3c). The coupling takes place at the interfaces, by communicating forces and displacements between the structure solver and flow solver. Convergence is assessed based on the $\mathrm{L}_{2}$-norm of the vectors containing the force and displacement residuals. A quasiNewton method ameliorates the convergence rate. This is implemented in the IQN-ILS algorithm (interface quasi-Newton with inverse Jacobian from a least-squares model), available in an in-house code called 'Tango' (Degroote et al., 2009). It was decided to increase the time step size to $1 \mathrm{~ms}$, facilitating both the convergence of the coupling iterations and the post-processing of displacement data with the discrete Fourier transform (DFT). The Nyquist frequency remains sufficiently high after this increase of the time increment, but a certain number of time steps spans a longer time window. As the computational load of the full FSI simulations is quite high, the number of FSI-time steps is limited to 1300.

\section{RESULTS AND DISCUSSION}

The numerical analysis described in Section 3 was performed for 3 different flow rates, summarized in Table 2. Velocity data is collected for a selection of points in the domain, at similar locations as during the experimental campaign. In Figure 4 those points are indicated by black dots. Their axial position correspond to the middle of the silicone part. They are equidistantly distributed between the central rod and the 6 walls of the hexagonal duct. 
Table 2. Overview of different flow rates and their respective pressure gradients.

\begin{tabular}{|l|l|}
\hline Flow rate $(1 / \mathrm{s})$ & Pressure gradient $(\mathrm{Pa} / \mathrm{m})$ \\
\hline 2.14 & 935.7 \\
2.68 & 1360.0 \\
3.48 & 2110.0 \\
\hline
\end{tabular}

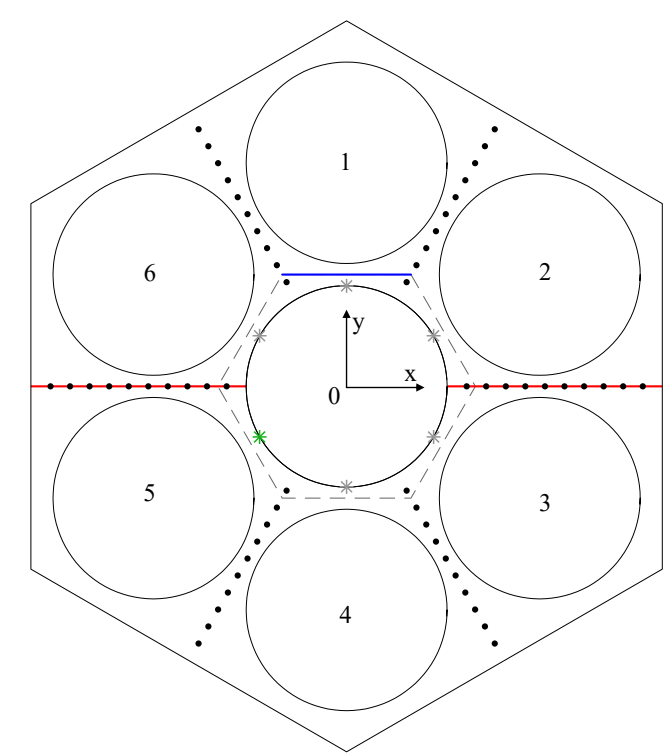

Figure 4. Schematic of the bundle. The positions at which velocity data is collected are indicated with black dots. The red and blue lines indicate two post-processing planes. The green asterisk indicates a point on the rod that faces a gap and from which displacement data is post-processed.

\subsection{Grid dependency}

A grid refinement study has been carried out for the CFD model. Only one direction of refinement was considered, namely the radial direction. Each mesh has thus 120 divisions in circumferential direction and 700 in axial direction. In addition to refinement, also additional grading is applied, to resolve the near-wall flow better. The study was performed for the case with a flow rate $\mathrm{Q}=2.14 \mathrm{l} / \mathrm{s}$. The meshes are named based on the number of faces in a cross-sectional plane and the number of axial divisions.

Because the same pressure gradient was used for each of the meshes, namely $935.7 \mathrm{~Pa} / \mathrm{m}$, it is to be expected that the flow rate will not be exactly the same, as can be seen in Table 3. After all the wall shear stress is very dependent on the boundary layer, which is resolved to a different degree for each of the meshes. The correctness of the wall shear force is not crucial for this research, as it is of little importance for the deformation of the silicone structure. In this work the main quantity of interest is the frequency of the flow pulsations. It can be seen that the number of structures in the domain is always around 13, which reinforces confidence in the chosen domain length.

Table 3. Overview of the different grids used for the grid refinement study.

\begin{tabular}{|l|l|l|l|l|l|}
\hline Name & Number of cells & Average wall $\mathrm{y}^{+}$ & Flow rate $(\mathrm{l} / \mathrm{s})$ & Bulk velocity $(\mathrm{m} / \mathrm{s})$ & Number of structures \\
\hline $6000 \times 700$ & 4200000 & 17.3 & 2.139 & 0.777 & $12-13$ \\
$12000 \times 700$ & 8400000 & 4.03 & 2.116 & 0.769 & 14 \\
$15600 \times 700$ & 10920000 & 2.91 & 2.137 & 0.777 & 14 \\
$19800 \times 700$ & 13860000 & 0.573 & 2.219 & 0.806 & 13 \\
\hline
\end{tabular}

To determine the frequency, the discrete Fourier transform of the velocity during the last 10000 time steps is computed. The definition used here is:

$$
A_{k}=\frac{1}{n} \sum_{m=0}^{n-1} a_{m} \exp \left(-2 \pi i \frac{m k}{n}\right), \quad k=0, \ldots, n-1
$$

$A_{k}$ is the $k^{\text {th }}$ Fourier coefficient, $a_{m}$ the $m^{\text {th }}$ element of the input vector, $\mathrm{n}$ the number of elements of the input vector and $i$ the imaginary unit. The input vector comprises the last 10000 time steps of the 20000 available time steps of the CFD results, thus after the flow has settled. When DFTs are reported in this text, the magnitude $\left|A_{k}\right|$ will be used. Considering the domain length, number of structures and bulk velocity (the flow rate divided by the through flow area), an order of magnitude of $10 \mathrm{~Hz}$ is expected for the vortex street. The velocity vectors are collected each time step at the locations indicated in Figure 4 . They are split in 3 components: the axial component, the radial component and the circumferential component. For each component a representative plot is shown in Figure 5. The location of the used data is picked based on the strength of the signal (also see Figure 6). From the spectra it is hard to pinpoint a single frequency as the dominant one, as the plots are quite spikey and the peaks shift a bit depending on the location 
and component that is considered. Nevertheless it is possible to situate the dominant frequency for every grid in the neighbourhood of $10 \mathrm{~Hz}$. It can also be seen in Figure 5 that all of the grids predict a secondary peak at twice the frequency of the dominant peak. This peak is rather prominent compared to the primary peak for the circumferential component in the neighbourhood of the rod.

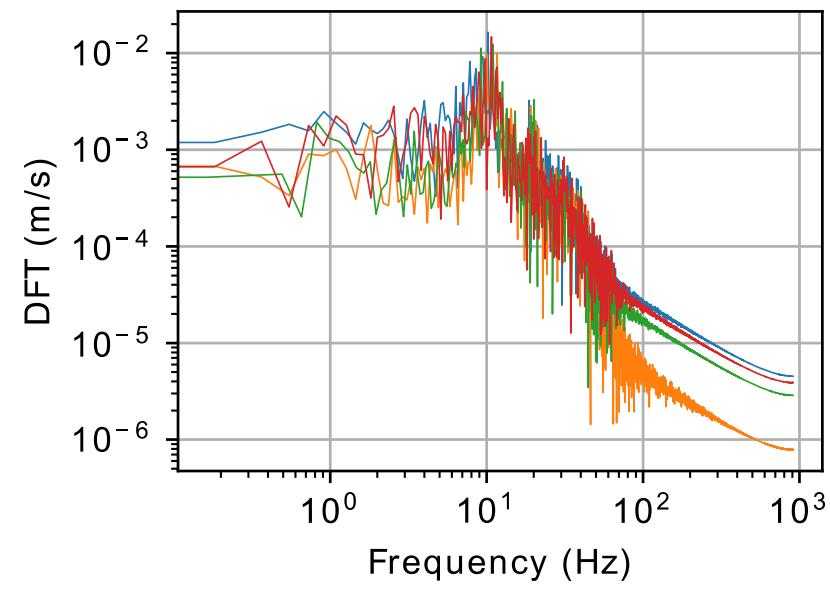

(a)

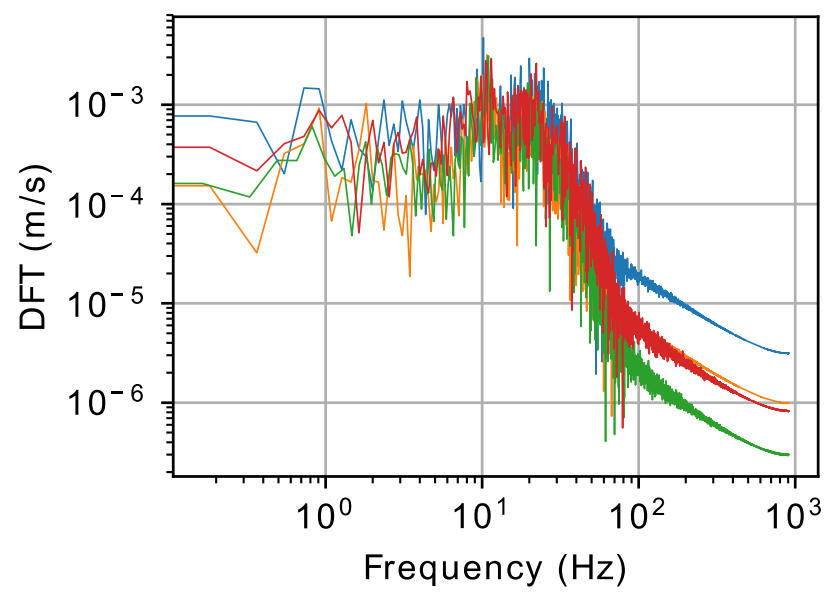

(c)

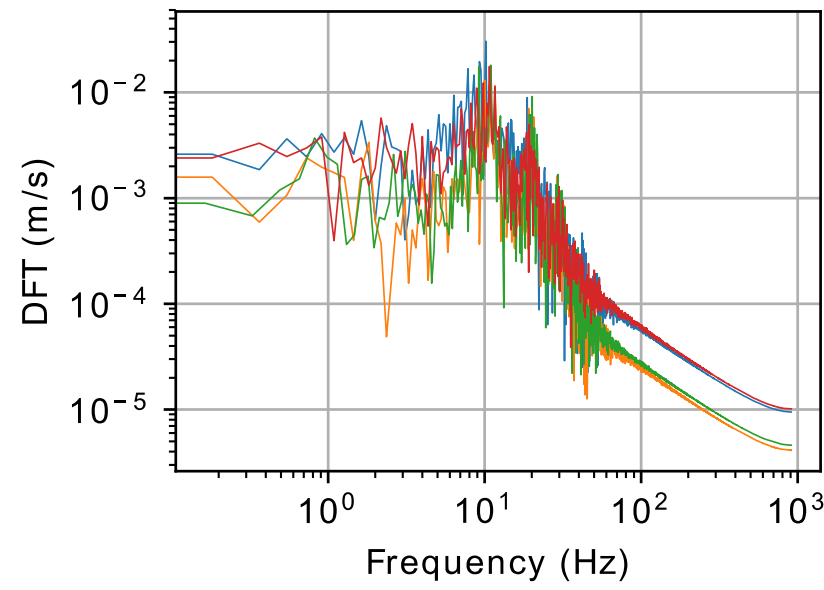

(b)

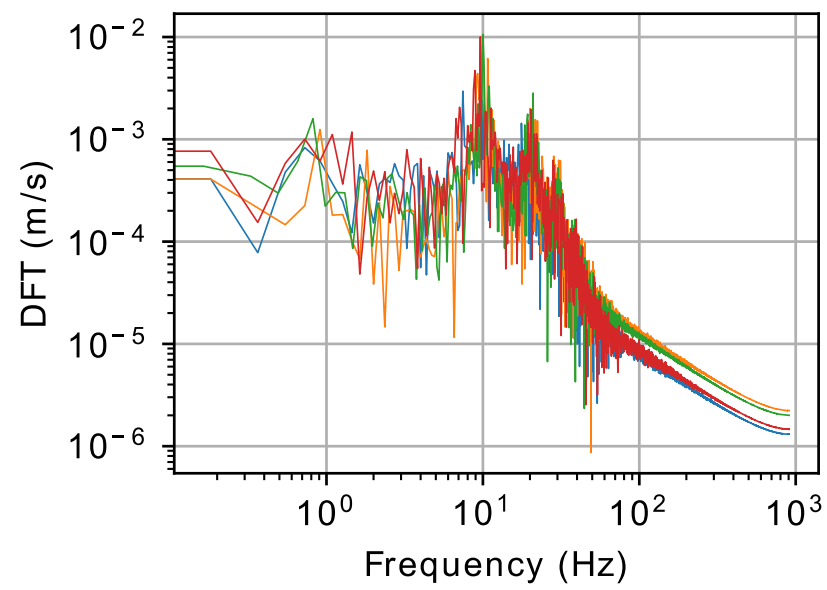

(d)

$6000 \times 700-12000 \times 700$

$15600 \times 700$

$19800 \times 700$

Figure 5. Magnitude of the DFT for the different grid resolutions of the (a) radial velocity component in the $5^{\text {th }}$ point from the central rod, (b) axial velocity component in the $8^{\text {th }}$ point from the central rod, (c) circumferential component in the $1^{\text {st }}$ point from and (d) circumferential component at the $8^{\text {th }}$ point from the central rod. The DFTs are generated from the data in between rod 4 and rod 5 (see Figure 4).

The analysis of the number of structures and frequency spectra is inconclusive. Therefore velocity profiles are shown in Figure 6. Again the time steps 10000 to 20000 are taken into account. For each component the root-mean-square value is calculated. As the 6 possible directions are geometrically equivalent, the arithmetic mean is taken of the results for those 6 directions. The values were normalized by the bulk velocity of the corresponding grid. It can be seen that the results lie quite close together, especially for the circumferential and axial component.

It was decided that the potential accuracy benefit of finer grid does not outweigh the increase in computational demand. This is especially the case because the benefit has not been unambiguously demonstrated and because of the considerable computational load a full FSI simulation brings about. Additionally, all grids overpredict the frequency found in the experiments, $7.77 \mathrm{~Hz}$. Based on the convection speed and the number of structures, a slightly lower frequency is present for the coarsest grid. It was therefore decided to use the $6000 \times 700$ mesh for the remainder of the research. 


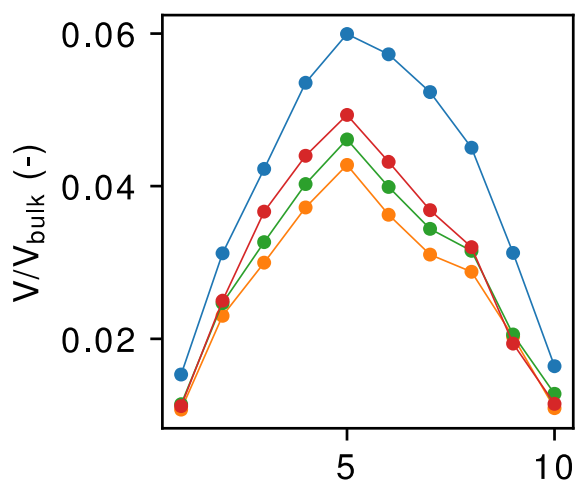

Radial

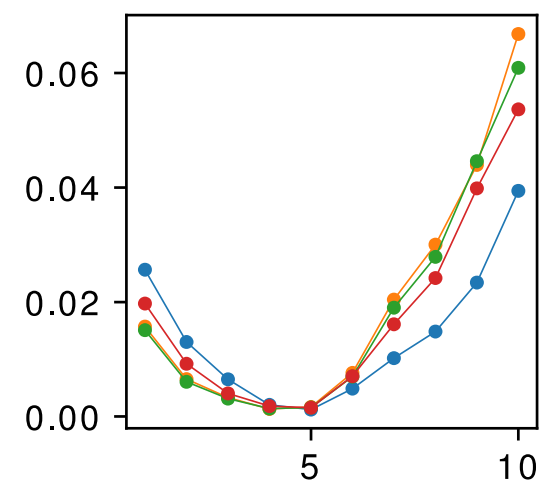

Circumferential

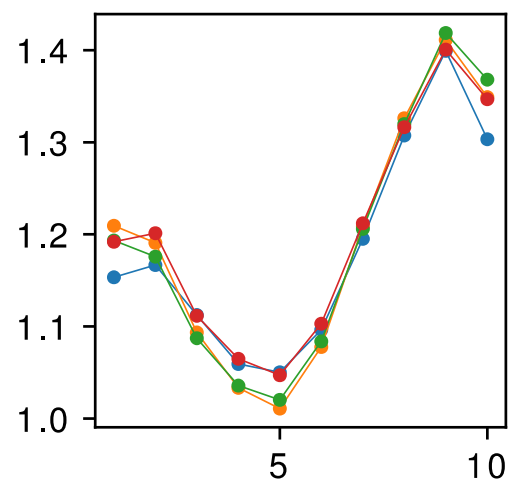

Axial

$\because 6000 \times 700 \div 12000 \times 700 \div 15600 \times 700 \quad \longrightarrow 19800 \times 700$

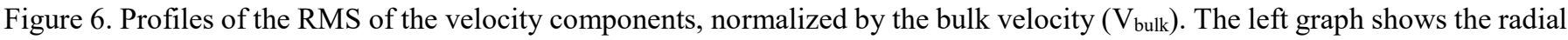
component, the central graph the circumferential component and the right graph the axial component. The results are averaged over the 6 different directions, indicated in Figure 4. The horizontal axis denotes the point at which the data was collected and the higher the number, the further away from the central rod.

Already some features of the flow become apparent. Firstly, by looking at the axial component, it can be seen that indeed a large velocity difference exists between the gaps and the subchannels. The axial velocity reaches a minimum around the middle of the gap ( $5^{\text {th }}$ point from the central rod), and two maxima. It can also be seen that this maximum is higher for an edge subchannel (next to the hexagonal duct), compared to a central subchannel (next to the central rod). This is because an edge subchannel has a larger local hydraulic diameter. Secondly, the radial component has a single maximum, also at the middle of the gap. The result of these radial fluctuations in this gap region is a considerable amount of flow crossing the gap. Towards the wall of the central rod and the edge of the channel, the RMS value drops to zero, as a radial oscillation is blocked by the walls. Finally, the opposite story holds for the circumferential component, which reaches a minimum at the middle of the gap. Due to the presence of the rods at both side of the gaps, there is no room for a circumferential oscillation over there. The RMS value of this component is high in the central and edge subchannels, achieving the highest value in the edge subchannel.

\subsection{Dominant frequency}

In this section the dominant frequency in the flow is determined from the results of the CFD simulations using two methods. When looking at a contour plot of the axial velocity (Figure 7), one can see that the slower moving fluid in the gap, oscillates back and forth between the central rod and the corner of the channel. This oscillation is not stationary, but is convected with the flow. An estimate of the wavelength can be made by counting the number of direction changes. Dividing the total length by half this number yields the (spatial) wavelength. Close to the centre of the figure, one wavelength seems to be stretched, as if a period is skipped. The pattern is indeed not entirely regular in these kind of flows, there is some chaos present, as was also found by Baratto et al. (2006). Another reason for the disturbed pattern could be that an integer number of wavelengths is forced in the domain. Missing periods have also been observed by Merzari and Ninokata (2011), which they hypothesised as being caused by interference effects between the vortex streets in different gaps. The number of wavelengths present is 12 to 13 for $Q=2.141 / \mathrm{s}$. This yields a wavelength of $0.077 \mathrm{~m}$ to $0.083 \mathrm{~m}$. The convection speed is estimated by comparing the flow pattern (Figure 7) at two different points of time, 100 time steps apart. The patterns at time step $\mathrm{N}$ is shifted in space for a distance s (corresponding to the grid size) and subtracted from the pattern at time step $\mathrm{N}+100$. The distance travelled by the pattern can then be estimated as being equal to $\mathrm{s}$ when the $\mathrm{L}_{2}$-norm of the difference is minimal. Dividing this distance by the elapsed time then yields the convection speed $\mathrm{V}_{\text {conv. }}$ It turned out that this convection speed was for all cases almost equal to the bulk velocity, which will be used instead throughout this work. For $\mathrm{Q}=2.14 \mathrm{l} / \mathrm{s}$ this velocity is $0.777 \mathrm{~m} / \mathrm{s}$. Based on the convection velocity and the wavelength, the frequency is estimated to be $9.32 \mathrm{~Hz}$ to $10.10 \mathrm{~Hz}$.

Another possibility is calculating the discrete Fourier transform. An illustrative example of the spectrum can be seen in Figure 8 , where the DFT magnitude of the radial velocity component in the middle of a gap ( $5^{\text {th }}$ point from the central rod) is shown. Also for the axial component this is plotted, here from data collected at the $8^{\text {th }}$ point from the central rod. These distance are chosen because the signals for the respective component and position are clearest (also see Figure 6). A peak can indeed be observed at around 10 Hz. Around this main frequencies also some sidebands are present. Often a second peak can be observed as well, as is also the case here. This peak has about twice the frequency of the first one. This phenomenon has also been observed by De Ridder et al. (2016b). From the DFT plots it is also clear that the time step was chosen sufficiently small, as the Nyquist frequency is much higher than the peak. Also the simulated time window is chosen appropriately, since the lowest resolved frequency is much lower than the peak. 
Figure 7. Contour plot of the axial velocity in a plane going through the gaps between the peripheral rods, at time step 20000 . This plane is indicated in red in Figure 4. The water flows from left to right.

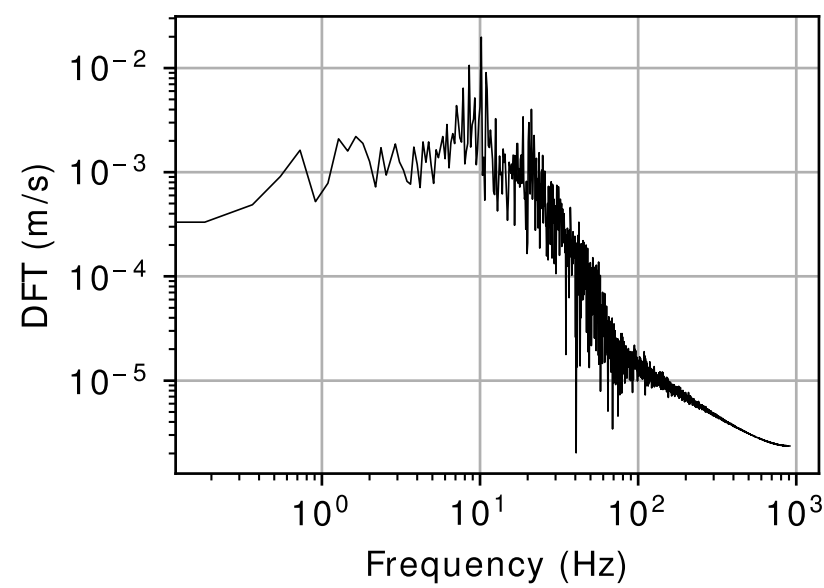

(a)

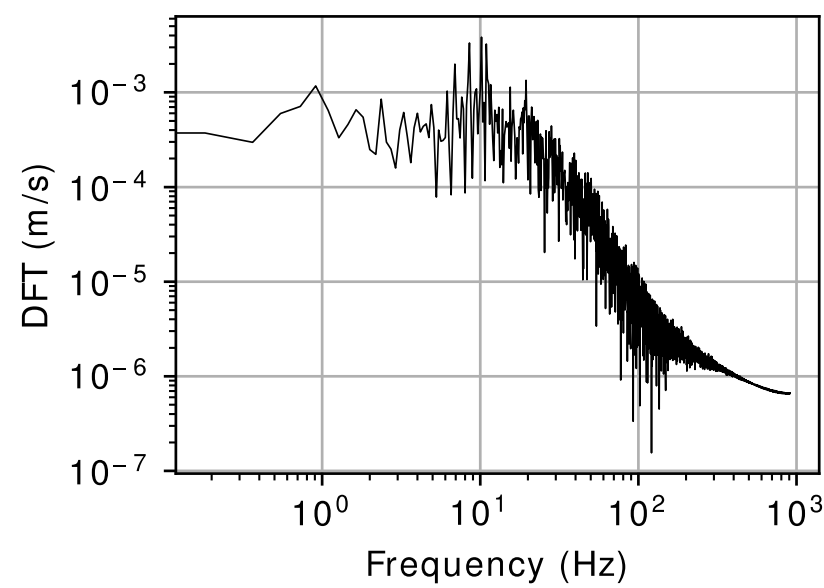

(b)

Figure 8. Magnitude of the Discrete Fourier transform of (a) the radial velocity component at the $5^{\text {th }}$ point from the central rod for $\mathrm{Q}=2.14 \mathrm{l} / \mathrm{s}$ and (b) the axial velocity component at the $8^{\text {th }}$ point from the central $\operatorname{rod}$ for $\mathrm{Q}=2.14 \mathrm{l} / \mathrm{s}$.

From both the flow field and frequency spectra it is concluded that the most important frequency is about $10 \mathrm{~Hz}$. This is close to the experimentally found frequency of $7.77 \mathrm{~Hz}$, yet not exactly the same.

The same analysis has been carried out for two other flow rates, $2.68 \mathrm{l} / \mathrm{s}$ and $3.48 \mathrm{l} / \mathrm{s}$. For the flow rate of $2.68 \mathrm{l} / \mathrm{s}$ again 12 to 13 structures were counted. Based on this the frequency is estimated to be 11.69 to $12.66 \mathrm{~Hz}$, the convection velocity being $0.974 \mathrm{~m} / \mathrm{s}$. A flow rate of $3.48 \mathrm{l} / \mathrm{s}$ corresponds to a $\mathrm{V}_{\text {conv }}$ of $1.263 \mathrm{~m} / \mathrm{s}$. With again 12 large structures in the domain, the frequency is around 15.16 Hz. These frequencies are confirmed by DFT graphs, depicted in Figure 9.

These results are summarized and compared with experiments in Table 4. In the experiments, the dominant frequency was determined by fitting a Gaussian bell curve to the frequency plots, as a broad peak rather than a sharp peak was present after postprocessing of the raw LDA data. One can see that the results do not exactly match. Nevertheless they are close, especially when one considers the standard deviation of the Gaussian curve used to fit to the experimental frequency plots.

Table 4. Numerically and experimentally found dominant frequency for each of the cases.

\begin{tabular}{|l|l|l|}
\hline Flow rate $(\mathrm{l} / \mathrm{s})$ & Dominant frequency simulation $(\mathrm{Hz})$ & Dominant frequency experiments $(\mathrm{Hz})$ \\
\hline 2.14 & $9.32-10.10$ & $7.77, \sigma=2.23$ \\
2.68 & $11.69-12.66$ & $8.80, \sigma=2.64$ \\
3.48 & 15.16 & $11.27, \sigma=3.55$ \\
\hline
\end{tabular}




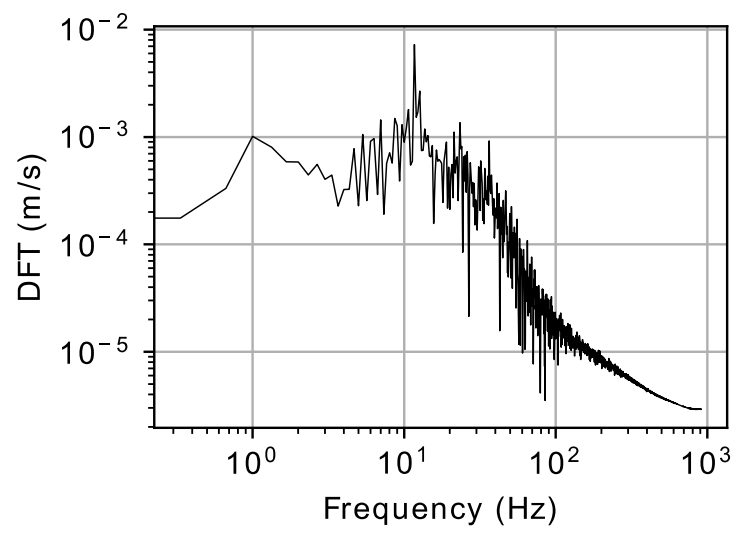

(a)

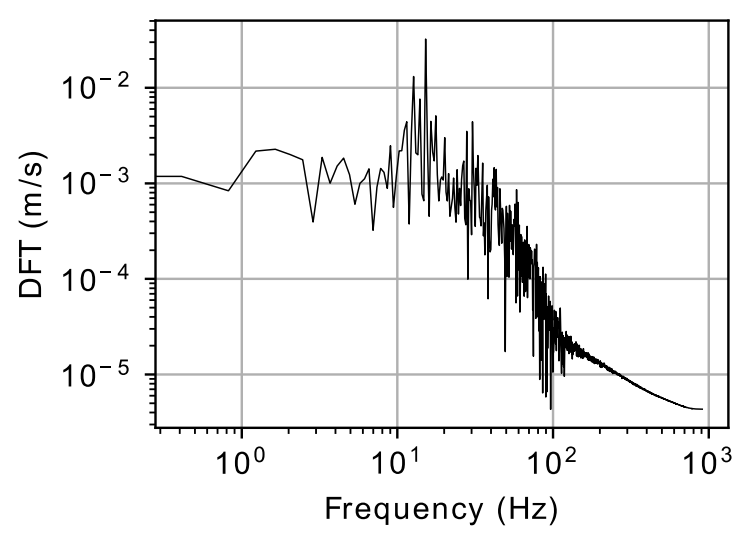

(b)

Figure 9. Magnitude of the DFT of the axial velocity at the $8^{\text {th }}$ point from the central rod for (a) $Q=2.681 / s$ and (b) $Q=3.481 / s$.

\subsection{Pressure field and structure of the flow field}

Before discussing the displacements, some attention is given to the pressure field incident on the central rod. This is the field that is communicated to the finite elements model and thus determines the vibration. The incident pressure field is displayed in Figure 10. One can see some zones of higher pressure and some zones of lower pressure. When looking at the top figure, of which the axis have equal scales, it can be seen that these zones form a kind of network. The bottom figure shows a detail, considering an axial section of only $100 \mathrm{~mm}$ length. This is the same length as the silicone rod. Zones of higher and lower pressure alternate each other, at both sides of the gap. The width of the pressure range is about $10 \mathrm{~Pa}$, so the forces involved are rather small.

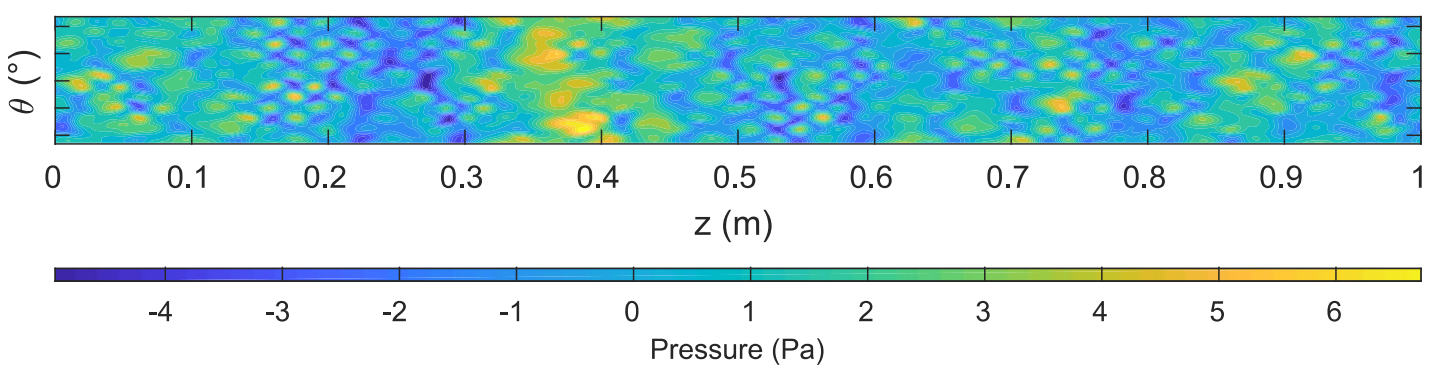

(a)

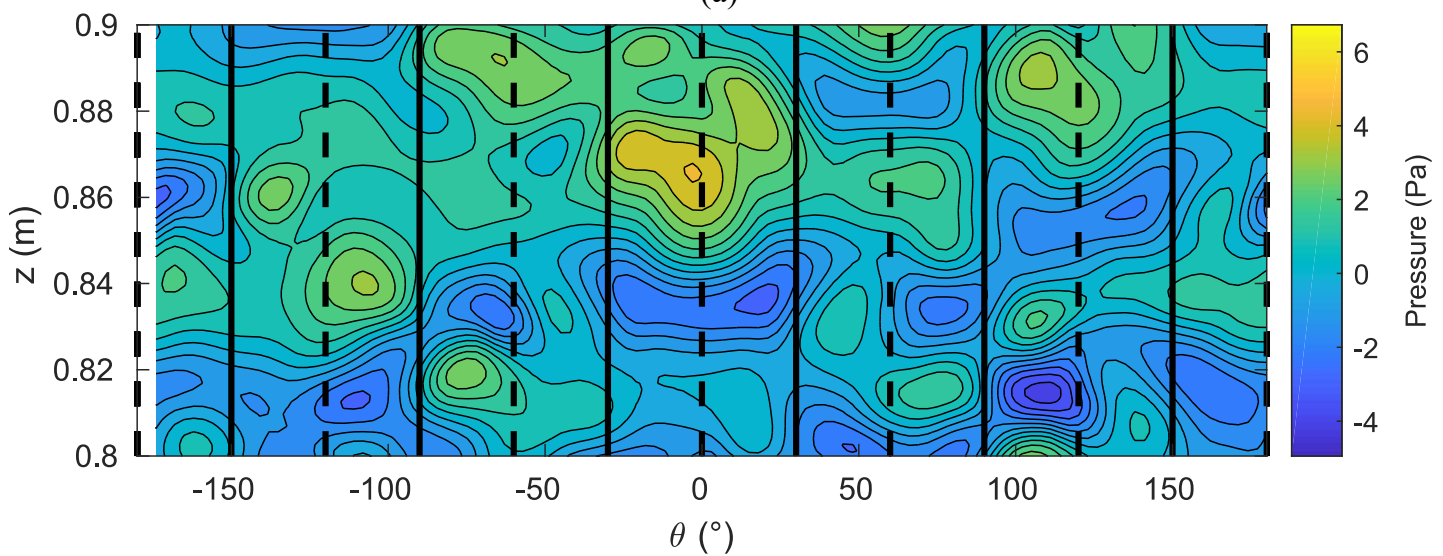

(b)

Figure 10. (a) Incident pressure field on the central rod with equal scale between the axes. (b) An axial section of $100 \mathrm{~mm}$ of the incident pressure field of the central rod. The dashed lines correspond to subchannels, the solid lines to gaps. Note that the axial coordinate is now on the vertical axis. The water flows in the positive $\mathrm{z}$-direction. The data is taken from the case with $\mathrm{Q}=2.14 \mathrm{l} / \mathrm{s}$. 


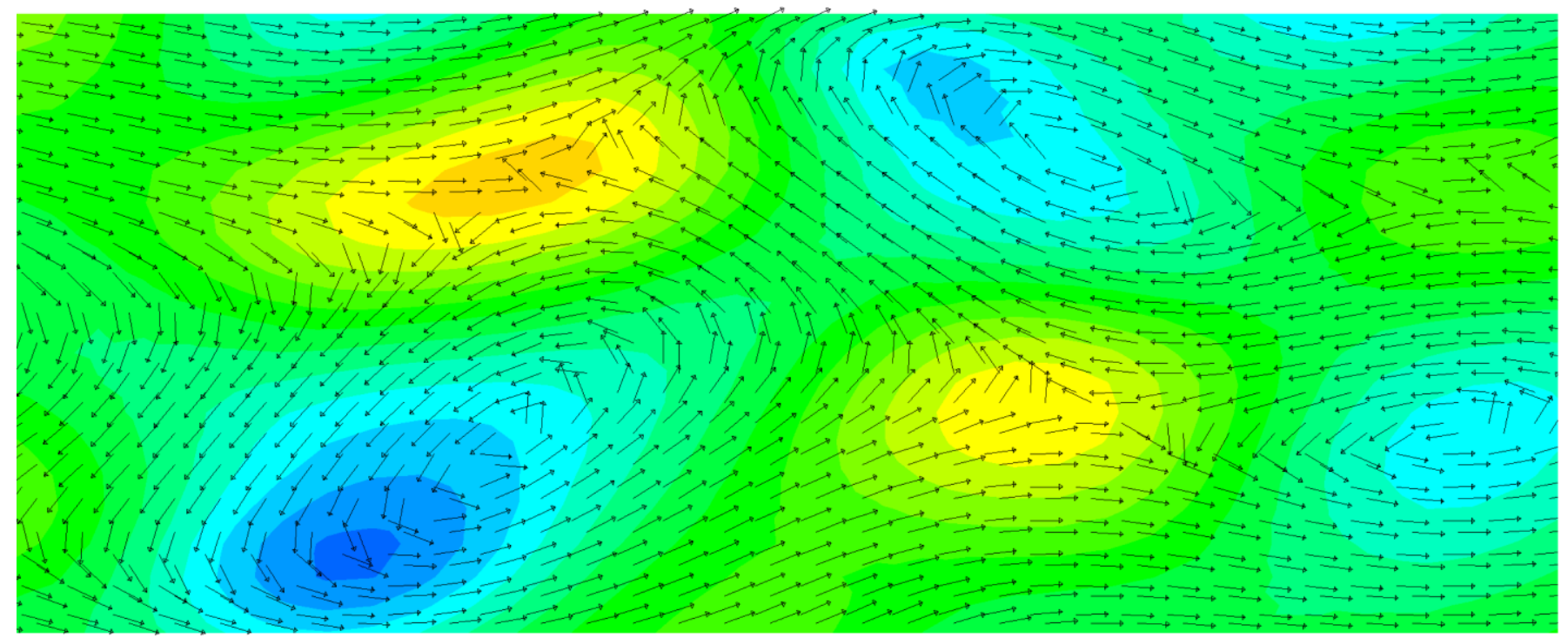

$-3.85 \mathrm{e}+00$
$\mathrm{~Pa}]$

Figure 11. Detail of the flow field in the plane indicated in blue in Figure 4, being the edge of the dashed hexagon. It concerns a contour plot of the pressure field with vectors of the relative velocity in overlay. The relative velocity is the velocity of which the bulk velocity is substracted from the axial component. The vectors are given constant length.

A more detailed view on the flow field is given in Figure 11. This plot is generated from data in a plane in between two rods, indicated in blue in Figure 4 as an edge of the small dashed hexagon. By reducing the axial component of the velocity with the bulk velocity, the vortical nature of the structures become clear. It can be seen that the low pressure zones are associated with circulating flow, providing the centripetal force to the vortices. The opposite happens at the high pressure zones, as these provide a force that curves away the streamlines. This structure of the flow field, alternating vortices at both sides of the gap, resembles well the one proposed by Meyer and Rehme (1994).

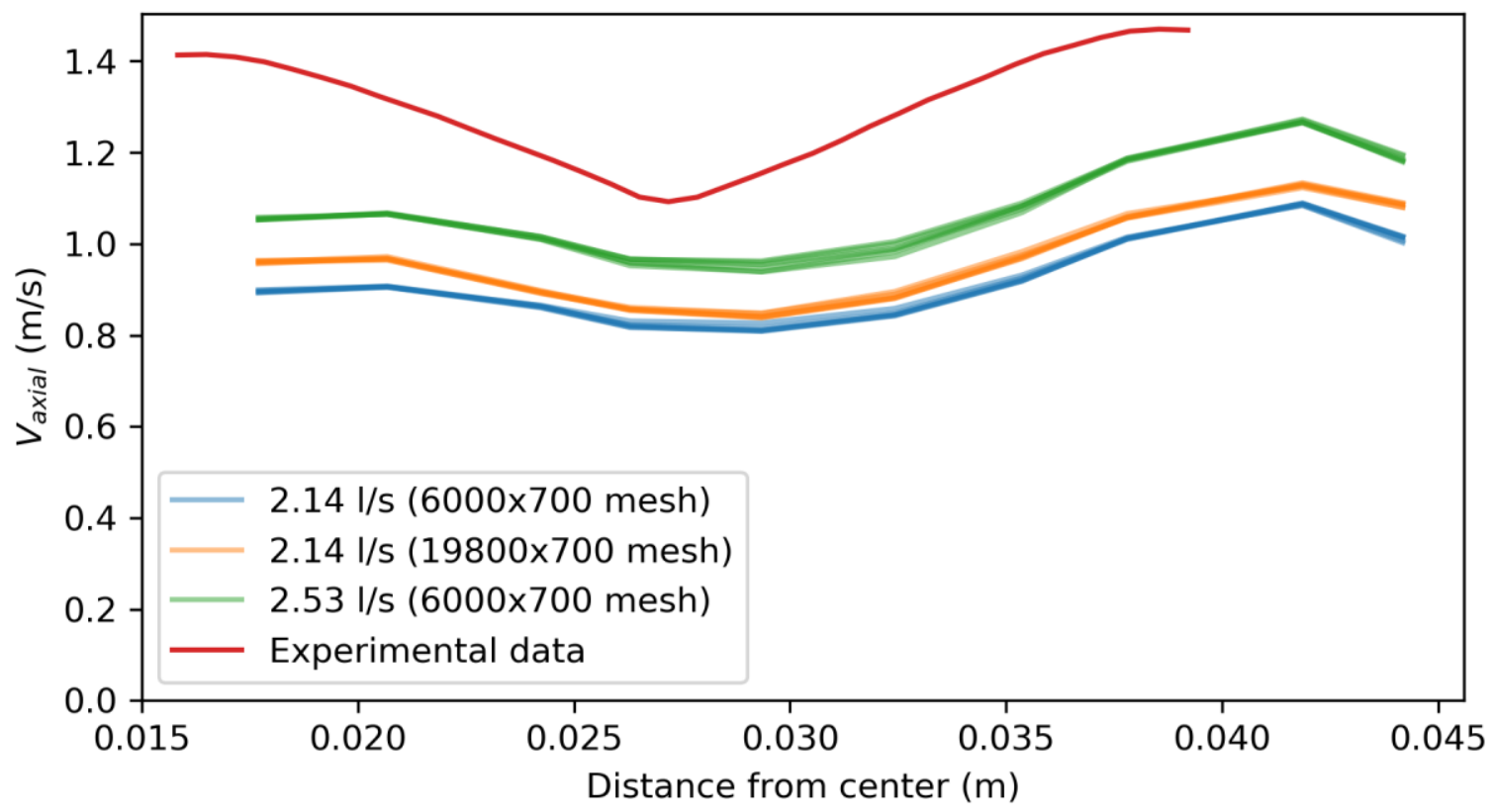

Figure 12. Comparison of velocity profiles for flowrates $2.14 \mathrm{l} / \mathrm{s}$ and $2.53 \mathrm{l} / \mathrm{s}$ with experimental data from LDA-measurements. The horizontal axis shows the distance from the centre. The CFD data corresponds to the black dots in Figure 4. The CFD profiles have been averaged out over the last 10000 time steps and are plotted for all 6 directions, such that the scatter is also shown.

Figure 12 shows a comparison between the velocity profiles along a line from the central rod to the wall of the channel (black dots in Figure 4). For a flow rate of $2.14 \mathrm{l} / \mathrm{s}$ the profile is plotted for the coarsest and finest mesh. It can be seen that there is a difference in magnitude, due to using the same pressure gradient as explained in Section 4.1. The shape is however the same. The experimentally obtained velocities are higher than the ones obtained using CFD. Measurements of the transparent section revealed 
that due to geometric tolerances the cross-section could be locally smaller, it is estimated that a difference in bulk velocity could be as high as $18 \%$. Therefore, a simulation with $18 \%$ higher flow rate $(2.53 \mathrm{l} / \mathrm{s})$ was performed. It can be seen that the shape of the profile appears similar to the other profiles obtained by CFD, but is still lower than the experimental profile.

The shape of the experimentally obtained profile appears more symmetric than in CFD. The velocity being higher at the edge subchannel is due to a larger local flow through area, this effect is more clearly present in the CFD data. The minimum in the gap region is also sharper and more pronounced for the experimental results. The reason for the differences is not known, but geometric tolerances possibly have a large effect.

\subsection{Displacements}

As explained in the Section 3, the CFD mesh has been adapted when switching from CFD simulations with rigid geometries to FSI with a flexible piece in the central rod. A snapshot of the deformed rod can be seen in Figure 13, the displacements are in the order of some micrometres. One can see that the deformation resembles the lower shell modes of a freely vibrating cylindrical shell.

Because only a small patch of the silicone wall was filmed during the experiments, only displacement information of a single point is captured and the position of the camera is such that this point is facing a gap. From the FSI simulations all displacement data is available. On the silicone rod there are 6 geometrically equivalent circumferential positions facing a gap, indicated by asterisk symbols in Figure 4. Data is extracted for each of these positions, in the middle of the axial range spanned by the silicone cylinder. These are plotted as a function of time, shown in Figure 14, for one of the 6 gap-facing points. It can be seen that the pattern is oscillatory, although not periodic. A main frequency is present, but also a lower-frequency modulation making the oscillations chaotic. A maximal displacement of about $6 \mu \mathrm{m}$ is achieved.

The frequency and amplitude of the displacement are compared to the values found in the experiments. For the frequency the Fourier transform is again used, while for the amplitude the root-mean-square is computed. The first 300 time steps are omitted in order to avoid possible influences of initialisation phenomena. Because the number of time steps for the post-processing of the FSI simulations is limited to 1000 , the frequency resolution is only $1 \mathrm{~Hz}$. The analysis is performed for all of the 3 flow rates. It is possible to compute 6 DFTs per simulation for each of the 6 geometrically similar points. The arithmetic average is taken over these 6 DFTs, to reduce the influence of outliers.

The result of the displacement is plotted in Figure 15 and a summary of the comparison can be found in Table 5. First, the simulation data are analysed. It can be seen from the DFTs that the magnitude rises with the flow rate. The same holds for the RMS values from the simulations. For the cases with flow rates $2.14 \mathrm{~m} / \mathrm{s}$ and $2.68 \mathrm{~m} / \mathrm{s}$ one would conclude that the displacement frequency matches closely with the frequencies found in the flow (tabulated in Table 4). For the flow rate of $3.48 \mathrm{~m} / \mathrm{s}$ this is not true, as the displacement frequency turns out to be lower than the frequency found in the flow. The reason for this is not clear. It has to be stressed that is difficult to pinpoint a single dominant flow pulsation frequency based on the DFT, since there is noise involved. Looking at flow visualisations can aid this. However, this method is also limited because only an instantaneous snapshot is taken into account.
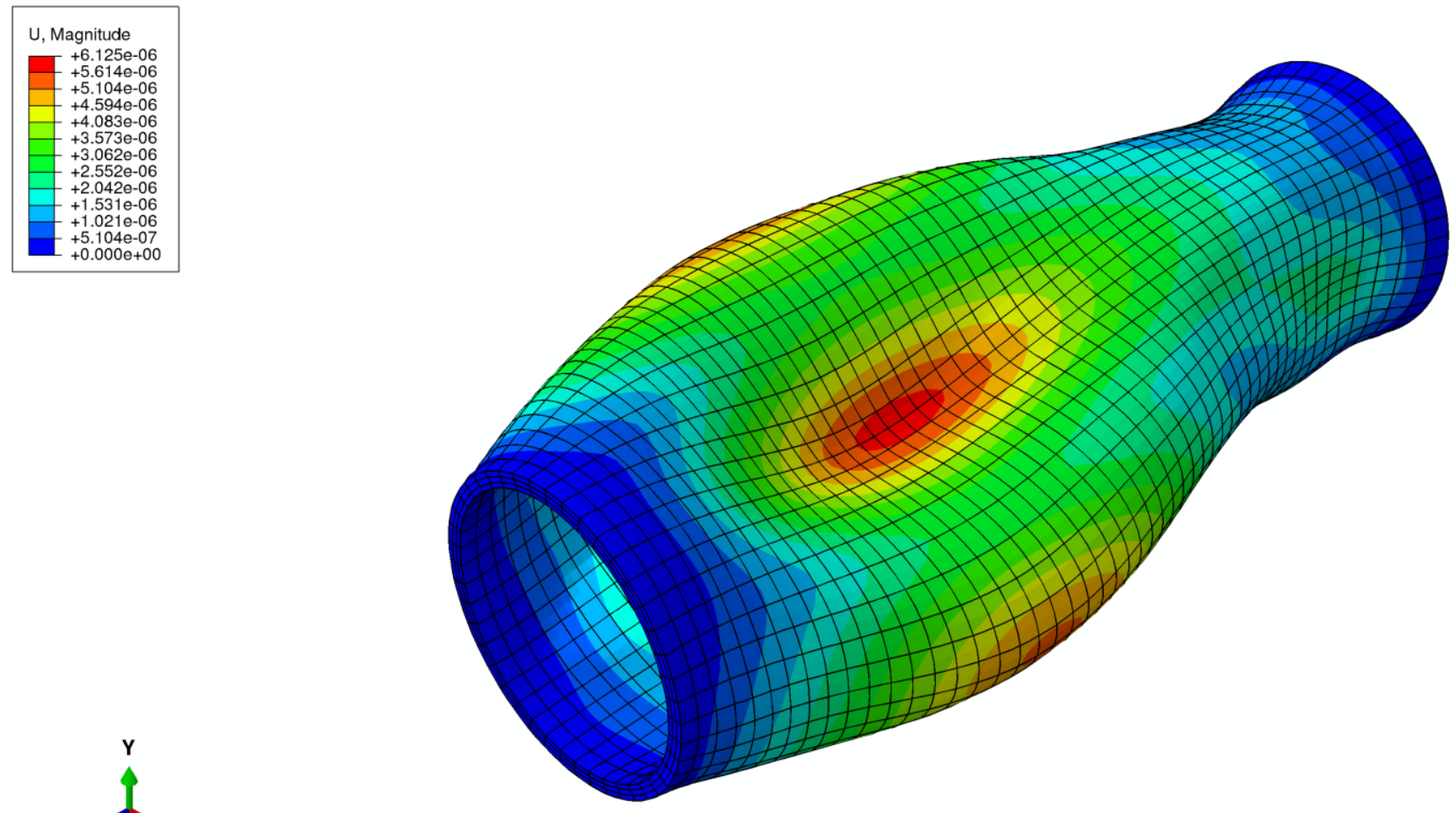

Figure 13. Displacement magnitude for the flexible segment of the central rod at time step 1250 for Q $=2.14$ 1/s. A uniform scale factor of 2000 was used. 


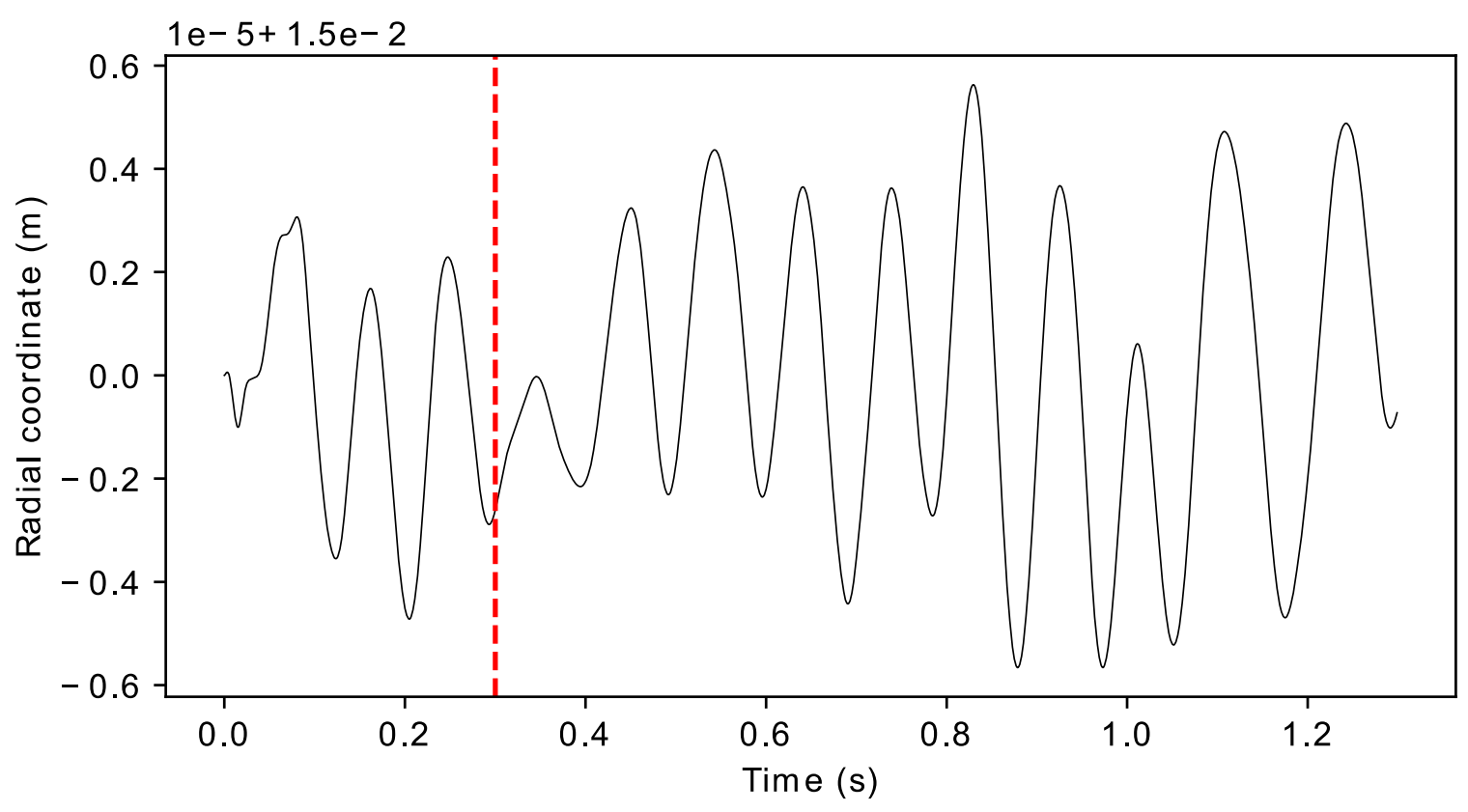

Figure 14. Displacement of a gap-facing point (green asterisk in Figure 4) on the surface of the silicone rod plotted versus time, for the case where the flow rate is $2.14 \mathrm{l} / \mathrm{s}$. Only data to the right of the red dashed line are used for the calculation of the DFT and RMS.

Second, the simulations and experiments are compared. A considerable discrepancy exists between the numerical and experimental results. The frequency differs with a factor 2 to 3 . In the experimental results the displacement frequency never matches the frequency found in the LDA data. In both the experimental and numerical results the frequency of the flow instability increases with the mass flow rate. This happens approximately linearly, as if 'frozen' structures are convected with the bulk flow. On the other hand, the frequency of the displacement does not rise steadily. The RMS values calculated from the simulations are always lower than the experimental ones. The difference is large for the case with $2.14 \mathrm{l} / \mathrm{s}$, but becomes rather small when $\mathrm{Q}=3.48 \mathrm{l} / \mathrm{s}$.

In summary it can be stated that the order of magnitude of the amplitudes is correctly predicted by the simulations. However, 3 simulations is too little to decipher a trend in the data. For the sake of completeness, the experimental results are depicted in Figure 16. It can be seen that the frequency of the displacement reaches a maximum for a certain flow rate, while the frequency of the axial velocity fluctuations rises linearly with the flow rate. It is hard to find a trend in the RMS data, also due to the variability of the amplitude as function of the flow rate.

Table 5. Comparison of the displacement data between the simulations and experiments.

\begin{tabular}{|l|l|l|l|l|}
\hline Flow rate (1/s) & Frequency simulation $(\mathrm{Hz})$ & Frequency experiments $(\mathrm{Hz})$ & RMS simulation $(\mathrm{m})$ & RMS experiments $(\mathrm{m})$ \\
\hline 2.14 & 10 & 3.77 & $2.79 \times 10^{-6}$ & $18.3 \times 10^{-6}$ \\
2.68 & 11 & 3.85 & $6.31 \times 10^{-6}$ & $11.7 \times 10^{-6}$ \\
3.48 & 11 & 3.49 & $7.90 \times 10^{-6}$ & $9.7 \times 10^{-6}$ \\
\hline
\end{tabular}




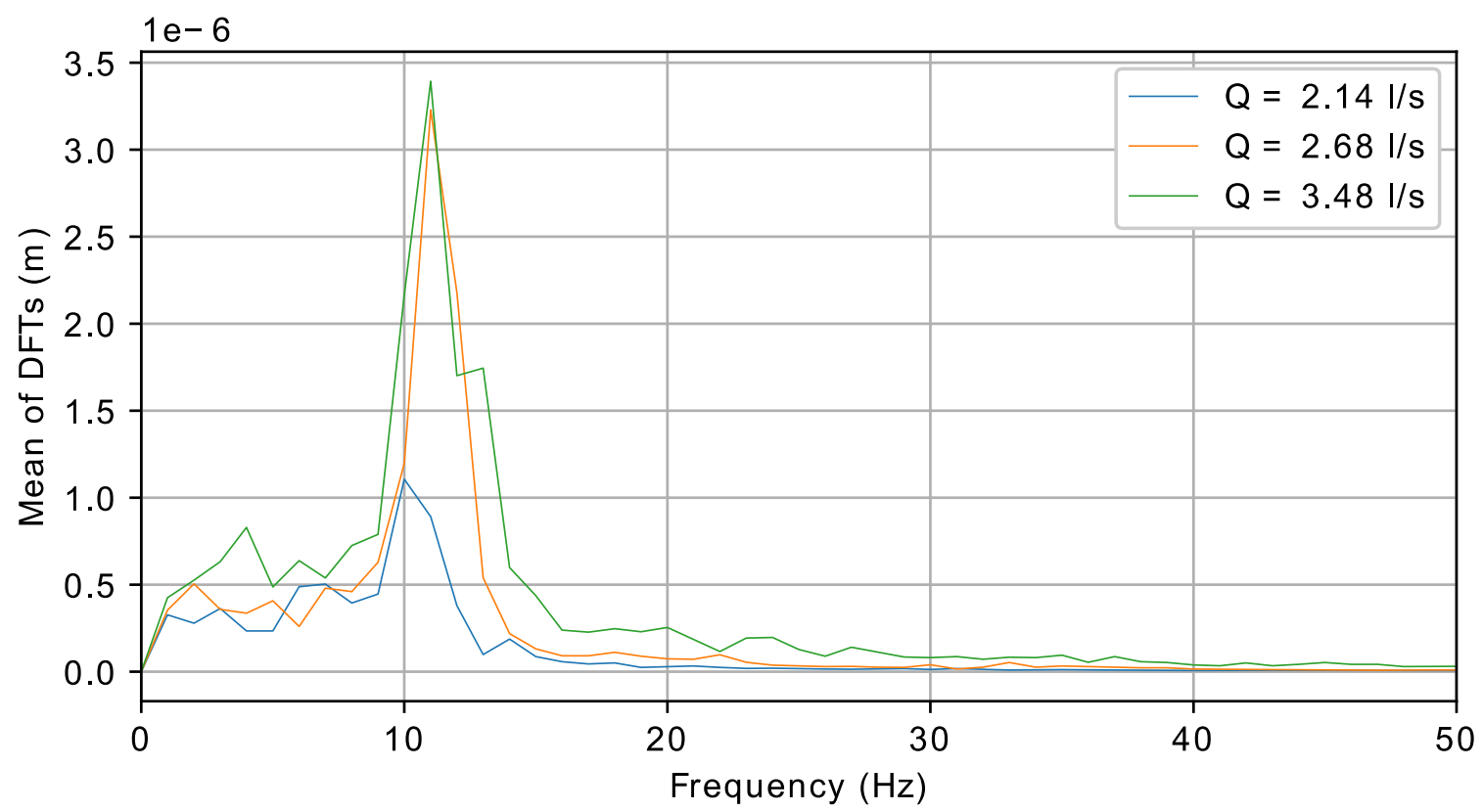

Figure 15. DFT of the displacement data, averaged over the 6 points (indicated with asterisks in Figure 4) on the surface of the silicone rod that face a gap.

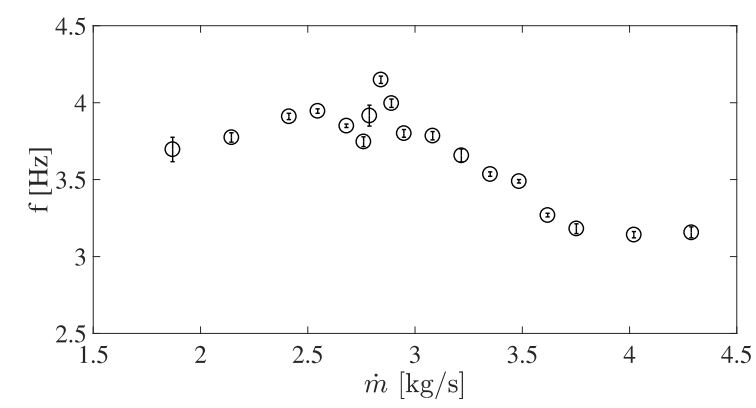

(a)

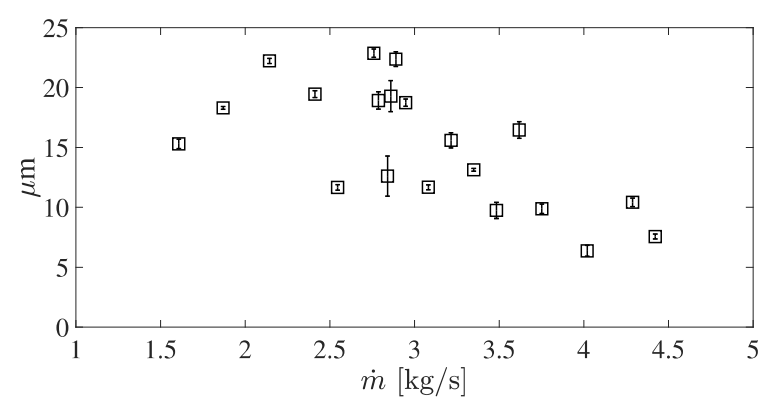

(b)

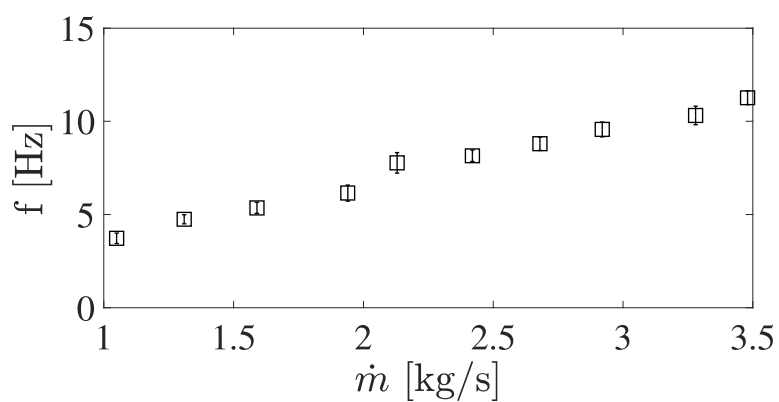

(c)

Figure 16. Experimental results: (a) the displacement frequency as a function of the flow rate, (b) the RMS value of the displacement and (c) the axial velocity fluctuation frequency. Data from Bertocchi et al. (2019).

From both the experimental results and numerical simulations questions arise how to classify this kind of vibration. Here the most important load component is attributed to large-scale vortices. The term vortex-induced vibration is however usually applied when the vibration of the structure matches the frequency of a vortex phenomenon, where the frequency rises linearly as a function of the mass flow rate. Only where lock-in occurs, this linearity is broken and the dominant flow frequency synchronizes with a resonance frequency of the structure, for a small range of mass-flow rates. The numerical data that has been collected in this work is not sufficient to either confirm or disprove this. Collecting data for more different flow rates could prove useful. The experimental results do not indicate any match between the frequency of the flow pulsations and structure, thus arguing against classifying them as vortexinduced. 


\subsection{Sensitivity of displacements to the geometry}

Because of the mismatch between experimental and numerical results, sources of uncertainty were sought. As some geometric uncertainty could be present in the experiment, a sensitivity study was performed. From the baseline case with a flow rate of $2.141 / \mathrm{s}$ two new cases were constructed. In the first case the length of the silicone rod has been decreased from $100 \mathrm{~mm}$ to $95 \mathrm{~mm}$. In the second case the thickness has been increased from $1.5 \mathrm{~mm}$ to $2.5 \mathrm{~mm}$, resulting in a stiffer rod. The results are presented in Table 6 .

Table 6. Results of the geometric parameter study.

\begin{tabular}{|l|l|l|l|l|}
\hline Flow rate $(\mathrm{l} / \mathrm{s})$ & Length silicone rod $(\mathrm{m})$ & Thickness silicone rod $(\mathrm{m})$ & Frequency $(\mathrm{Hz})$ & RMS $(\mathrm{m})$ \\
\hline 2.14 & $100 \times 10^{-3}$ & $1.5 \times 10^{-3}$ & 10 & $2.79 \times 10^{-6}$ \\
2.14 & $95 \times 10^{-3}$ & $1.5 \times 10^{-3}$ & 10 & $2.84 \times 10^{-6}$ \\
2.14 & $100 \times 10^{-3}$ & $2.5 \times 10^{-3}$ & $4 / 10 / 17$ & $6.82 \times 10^{-7}$ \\
\hline
\end{tabular}

The sensitivity to the length is very low, as can be observed from the table. The frequency and RMS are (almost) the same, for this resolution of frequencies. The thicker rod behaves differently. The amplitude decreased by a factor of 4 . This decrease was anticipated, as the stiffness has increased. The main frequency peak is situated at $10 \mathrm{~Hz}$, the same frequency as the flow pulsations. Interestingly, some other peaks appear in the DFT plot. The largest one is situated at $4 \mathrm{~Hz}$, which is closer to the experimentally measured frequency. The second largest one is at $17 \mathrm{~Hz}$. A higher frequency is what one expects when the stiffness increases. The DFT plot is shown in Figure 17 and the displacement of one of the points as a function of time in Figure 18. Upon inspection of the time data, it becomes clear that the other frequency peaks are due to a different pattern of the displacement. It can indeed be observed that not a single temporal period dominates the plot in Figure 18, but that other periods are present as well.

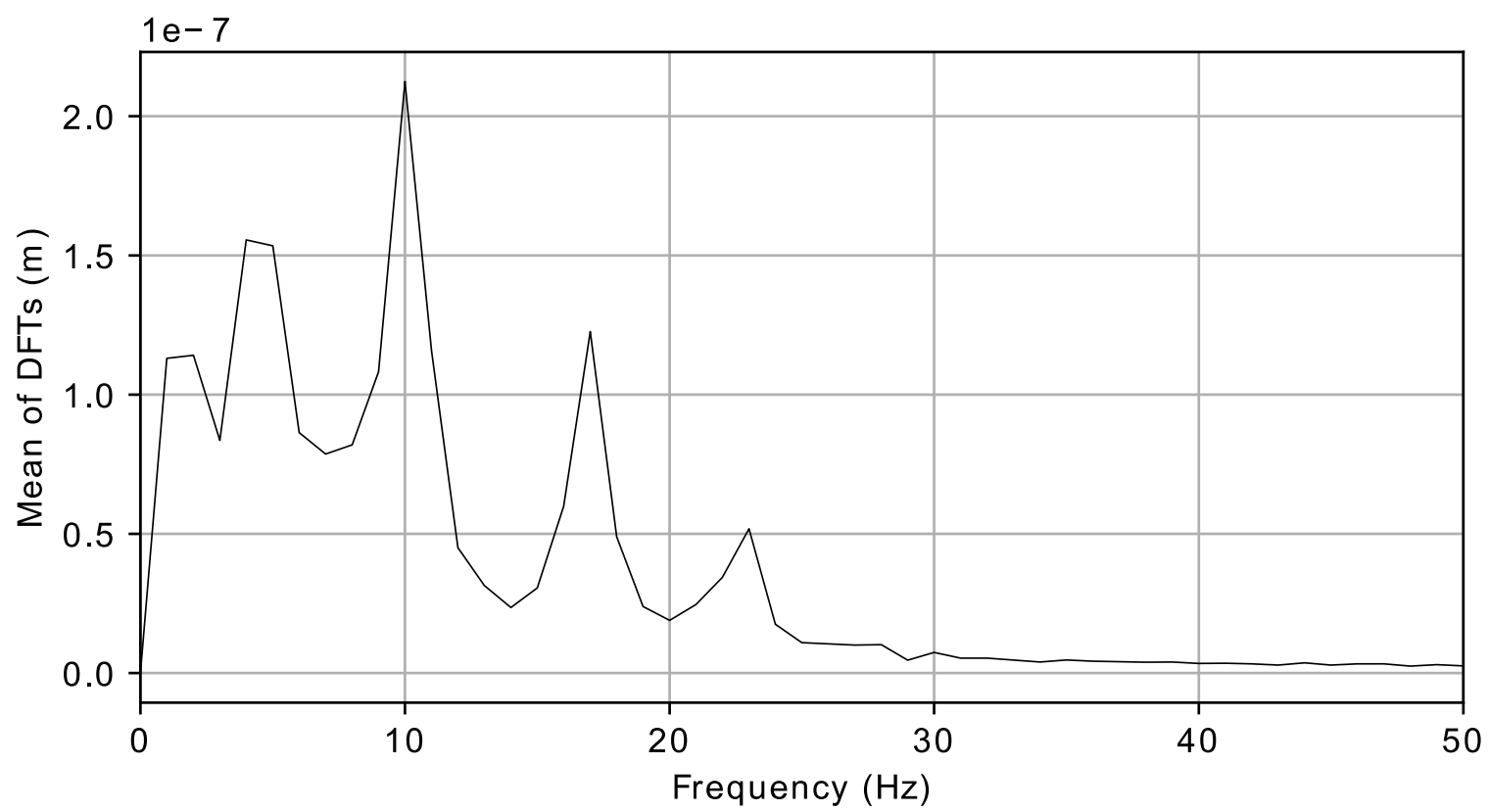

Figure 17. DFT of the displacement for the simulation with increased rod thickness, averaged over the 6 gap-facing points (asterisks in Figure 4). Peaks can be seen at 4-5 Hz, $10 \mathrm{~Hz}, 17 \mathrm{~Hz}$ and $23 \mathrm{~Hz}$. 


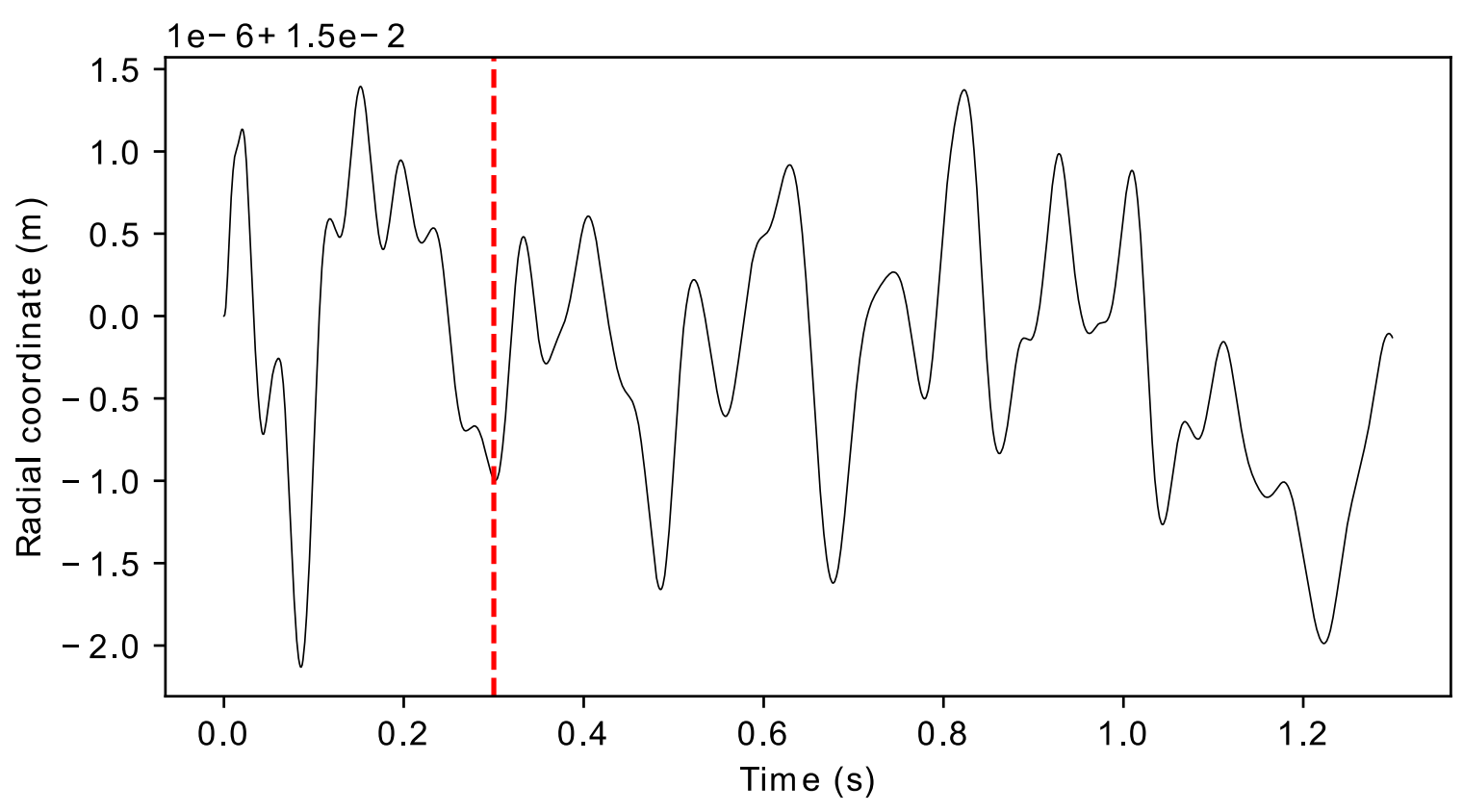

Figure 18. Displacement of a point sitting (green asterisk in Figure 4) on the wall of the silicone rod for increased rod thickness. Radial coordinate as a function of time.

\subsection{Sensitivity of displacement to the flow rate}

The rods surrounding the silicone tube are not made of steel, contrary to other axial locations, because they have to be transparent. This could however cause some additional geometrical uncertainty. As briefly mentioned in Section 4.3 it is estimated that the possibility exists that the through flow section is constricted at the height of the silicone rod, such that the bulk velocity can be up to $18 \%$ higher than nominal. As an attempt to assess the consequences of such a constriction, the flow rate has been increased with $18 \%$, from $2.14 \mathrm{l} / \mathrm{s}$ to $2.53 \mathrm{l} / \mathrm{s}$. It would be rather difficult to take such a constriction into account in the geometry of the numerical domain, as it concerns tolerances (thus not conserving the shape of the rods) that are hard to measure. The results are tabulated in Table 7. There are 13 to 14 wavelengths present, resulting in a frequency estimate of 11.96 to $12.88 \mathrm{~Hz}$, considering the convection velocity of $0.920 \mathrm{~m} / \mathrm{s}$. A value around $12 \mathrm{~Hz}$ is indeed confirmed upon inspection of the Fourier transform. Note that this deviates from the linear trend, as a lower frequency was observed for the case with a flow rate of $2.68 \mathrm{l} / \mathrm{s}$. This could be attributed to the uncertainty present in the frequency, possibly caused by forcing an integer number of wavelengths in a fixed-length domain. Again the frequency of the displacement matches the frequency of the flow pulsations.

Table 7. Results of the numerical sensitivity study towards the flow rate.

\begin{tabular}{|l|l|l|l|}
\hline Flow rate $(\mathrm{l} / \mathrm{s})$ & Frequency flow pulsations $(\mathrm{Hz})$ & Frequency displacement $(\mathrm{Hz})$ & RMS displacement $(\mathrm{m})$ \\
\hline 2.14 & 10 & 10 & $2.79 \times 10^{-6}$ \\
2.53 & $11.96-12.88$ & 12 & $6.49 \times 10^{-6}$ \\
\hline
\end{tabular}

A small constriction near the silicone rod could cause an intensification of the flow pulsations, which possibly partly explains why a higher displacement amplitude is observed in the experiments. This intensification could be caused by change in P/D ratio, of which the fluctuation strength is a function (De Ridder et al., 2016b).

The higher amplitude is in favor of the hypothesis that a constriction could be present, however in that case also a higher frequency would be expected. So following the hypothesis, all numerically found frequencies should be scaled up, increasing the mismatch with the experimental ones. This hypothesis alone is thus insufficient as an explanation for the mismatch and an error will persist, either in frequency or amplitude.

Other sources of uncertainty could be the elastic modulus E and Poisson's ratio $v$. The production tolerance is unknown and no mechanical test data is available for the silicone used in the part. Also the way that the inner fluid is taken into account could be partially responsible for the mismatch. In the structural model, only the silicone part is considered flexible. In reality however, this silicone part is mounted on two long steel rods. These are neglected in the finite elements model, since clamped boundary conditions were used. It is considered possible that these steel rods impose some other modes of vibration, appearing in the experiments but not covered by the simulations. 
An effort was made to create an accurate model for axial rod bundle flows displaying coherent structures and their resulting vibrations. The numerical results were validated against experimental results. The expected flow phenomena were observed. The displacement turned out to resemble the lower modes of a vibrating cylindrical shell.

The frequency of the flow pulsations was slightly higher than the one found experimentally. Similar to the experiments, it showed a linearly increasing trend, up to some uncertainty. It could be useful to investigate a wider range of flow rates to confirm this result. This is a chaotic flow phenomenon, multiple frequencies can be found and it is difficult to pinpoint a single one. The agreement with the experiments is however reasonable.

The displacement frequency was consistently higher in the simulations, compared to the measurements. Often a match between the frequency of the displacement and the flow pulsation was observed in the simulation. This is not the case for the experimental results, where the displacement frequency was lower than the flow pulsation frequency. The simulations predict a lower amplitude for the displacement of the rod, mainly for the lower flow rates. A geometrical sensitivity study was performed to investigate possible sources of the discrepancy. It turned out that uncertainty about the length of the flexible rod would have limited influence, but that the thickness has a large effect. Another possible source of uncertainty is a constricted through flow area in the neighbourhood of the silicone rod. This possibly causes an intensification of the flow pulsations, resulting in higher amplitudes. As this constriction is not acknowledged by the model and is hard to implement, it could not be pointed out as the origin of the discrepancy. It was also argued that it has no potential of being the complete explanation. The reason of the mismatch remains unknown. Some uncertainty is known to be present, but also some unacknowledged variability may have had influence.

The model performs qualitatively well, but some discrepancies persist. As complex phenomena are acting, constructing an accurate model is a challenging task. Clamped-clamped boundary conditions may have been too constraining, as the steel rods could impose some other vibrational modes. An uncertainty quantification may be useful to assess the probability that differences are caused by variability. Also more complex models, like LES, could be used on the simplified geometry or a more realistic representation, to better characterize the physics and to assess if any important physics are missed.

\section{ACKNOWLEDGMENTS}

This work was performed in the framework of the H2020 SESAME Project. It has received funding from the Euratom research and training program 2014-2018 under grant agreement No 654935 (SESAME).

The computational resources (Stevin Supercomputer Infrastructure) and services used in this work were provided by the VSC (Flemish Supercomputer Centre), funded by Ghent University, FWO and the Flemish Government - department EWI.

A special thanks to the recently deceased Prof. dr. ir. Jan Vierendeels for his guidance. His work yielded an important contribution to the field of fluid mechanics.

\section{REFERENCES}

Bertocchi F., Rohde M. and Kloosterman J.L., 2018. LDA measurements of coherent flow structures and cross-flow across the gap of a compound channel with two half-rods. Nucl. Eng. Des., vol. 326, pp. 17-30.

Bertocchi F., Rohde M., De Santis D., Shams A., Dolfen H., Degroote J., and Vierendeels J., 2019. Fluid-structure interaction of a 7-rods bundle: benchmarking numerical simulations with experimental data. Proc. SESAME Final International Workshop, Petten, The Netherlands, 19-21 March 2019.

Baratto F., Bailey. S and Tavoularis, S., 2006. Measurements of frequencies and spatial correlations of coherent structures in rod bundle flows. Nucl. Eng. Des., vol. 236, pp. 1830-1837.

Chang D. and Tavoularis S., 2005. Unsteady Numerical Simulations of Turbulence and Coherent Structures in Axial Flow Near a Narrow Gap. J. Fluids Eng., vol. 127, pp. 458-466.

Chang D. and Tavoularis S., 2006. Convective Heat Transfer in Turbulent Flow Near a Gap. J. Heat Transfer, vol. 128, pp. 701-708.

Chang D. and Tavoularis S., 2007. Numerical simulation of turbulent flow in a 37-rod bundle. Nucl. Eng. Des., vol. 237, pp. 575590.

Choueiri, G. and Tavoularis, S., 2014. Experimental investigation of flow development and gap vortex street in an eccentric annular channel. Part 1. Overview of the flow structure. J. Fluid Mech., vol. 752, pp. 521-542.

Choueiri, G. and Tavoularis, S., 2015. Experimental investigation of flow development and gap vortex street in an eccentric annular channel. Part 2. Effects of inlet conditions, diameter ratio, eccentricity and Reynolds number. J. Fluid Mech., vol. 768, pp. 294315.

Curling L. and Païdoussis M.P., 1992a. Measurements and characterization of wall-pressure fluctuations on cylinders in a bundle in turbulent axial flow: Part 1: spectral characteristics. J. Sound Vib., vol. 157, pp. 405-433.

Curling L. and Païdoussis M.P., 1992b. Measurements and characterization of wall-pressure fluctuations on cylinders in a bundle in turbulent axial flow: Part 2: temporal characteristics. J. Sound Vib., vol. 157, pp. 435-449.

Degroote J., Bathe K.-J. and Vierendeels J., 2009. Performance of a new partitioned procedure versus a monolithic procedure in fluid-structure interaction. Comput. Struct., vol 87, pp. 793-801.

De Moerloose L., Aerts P., De Ridder J., Vierendeels J. and Degroote J., 2018. Numerical investigation of large-scale vortices in an array of cylinders in axial flow. J. Fluids Struct., vol. 78, pp. 294-315.

De Ridder J., Degroote J., Van Tichelen K., Schuurmans P. and Vierendeels J., 2016a. Predicting turbulence-induced vibration in axial annular flow by means of large-eddy simulations. J. Fluids Struct., vol. 61, pp. 115-131. 
De Ridder J., Degroote J., Vierendeels J. and Van Tichelen K., 2016b. Vortex-induced vibrations by axial flow in a bundle of cylinders. Proc. $11^{\text {th }}$ Conf. on Flow-Induced Vibrations and Noise, The Hague, The Netherlands, 4-6 July 2016.

Donea J., Huerta A., Ponthot J.-P. and Rodriguez-Ferran A., 2004. Encyclopedia of Computational Mechanics, volume 1: Fundamentals, chapter 14 Arbitrary Lagrangian-Eulerian Methods, pp. 1-25. John Wiley \& Sons.

Guellouz M. and Tavoularis S., 2000a. The structure of turbulent flow in a rectangular channel containing a cylindrical rod - Part 1: Reynolds-averaged measurements. Exp. Therm. Fluid Sci., vol. 23, pp. 59-71.

Guellouz M. and Tavoularis S., 2000b. The structure of turbulent flow in a rectangular channel containing a cylindrical rod - Part 2: phase-averaged measurements. Exp. Therm. Fluid Sci., vol. 23, pp. 75-91.

Hooper J. and Rehme, K., 1984. Large-scale structural effects in developed turbulent flow through closely-spaced rod arrays. J. Fluid Mech., vol. 145, pp. 305-377.

Menter F.R., 1994. Two-equation eddy-viscosity turbulence models for engineering applications. Ind. Eng. Chem. Res., vol. 32, pp. 1598-1605.

Merzari E. and Ninokata H., 2011. Proper orthogonal decomposition of the flow in a tight lattice rod-bundle. Nucl. Eng. Des., vol. 241, pp. 4621-4632.

Meyer L. and Rehme K., 1994. Large-Scale Turbulence Phenomena in Compound Rectangular Channels. Exp. Therm. Fluid Sci., vol. 8, pp. 286-304.

Meyer L., 2010. From discovery to recognition of periodic large scale vortices in rod bundles as a source of natural mixing between subchannels - a review. Nucl. Eng. Des., vol. 240, pp. 1575-1588.

Monson H.H, 1996. Statistical Digital Signal Processing and Modeling. John Wiley \& Sons.

Païdoussis M.P. and Curling L., 1985. An analytical model for vibration of clusters of flexible cylinders in turbulent axial flow. J. Sound Vib., vol. 98, pp. 493-517.

Païdoussis M.P., 2004. Fluid-Structure Interactions - Slender Structures and Axial Flow: Volume 2. Academic Press.

Tavoularis S., 2011. Rod bundle vortex networks, gap vortex streets, and gap instability: A nomenclature and some comments on available methodologies. Nucl. Eng. Des., vol. 241, pp. 2624-2626. 